\title{
NONLINEAR OBSERVERS IN REFLEXIVE BANACH SPACES
}

\author{
Jean-François Couchouron ${ }^{1}$ and P. Ligarius ${ }^{2}$
}

\begin{abstract}
On an arbitrary reflexive Banach space, we build asymptotic observers for an abstract class of nonlinear control systems with possible compact outputs. An important part of this paper is devoted to various examples, where we discuss the existence of persistent inputs which make the system observable. These results make a wide generalization to a nonlinear framework of previous works on the observation problem in infinite dimension (see $[11,18,22,26,27,38,40]$ and other references therein).
\end{abstract}

Mathematics Subject Classification. 47H020, 47H06, 93B07, 93C20, 93C25.

Received December 3, 2001. Revised January 10, 2002.

\section{INTRODUCTION AND PRESENTATION OF THE RESUlTS}

We consider the following general nonlinear system on an arbitrary reflexive Banach space $X$,

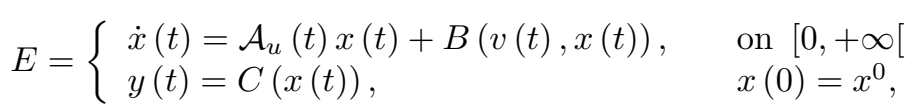

where $x^{0} \in X$ and the controls $(u, v)$ belong to $\mathcal{U} \times \mathcal{V} \subset \mathbb{L}^{\infty}\left(\left[0,+\infty\left[, \mathbb{R}^{m} \times \mathbb{R}^{q}\right)\right.\right.$. Here, $\left(\mathcal{A}_{u}(t)\right)_{t>0}$ represents a family of unbounded dissipative linear operators; the bounded dissipative nonlinear operator $B$ is defined on $\mathbb{R}^{q} \times X$; the bounded linear (observation) operator $C$ maps from $X$ to $Y$, where $Y$ is a finite or infinite dimensional (observation) normed space $\left(Y=\mathbb{R}^{p}, p<+\infty\right.$, physically makes sense). The precise assumptions on these operators are given in Section 4.

In this abstract framework we are concerned with the dynamical state estimate of the system $E$ by means of the output $y$ and inputs $(u, v)$. In this goal, for a given solution $x$ of the system $E$, we construct an auxiliary system $\widehat{E}$ with inputs $(u, v ; y)$ and output $\widehat{y}=C \hat{x}$; the solution $\hat{x}$ of $\widehat{E}$ is expected to "estimate" asymptotically the state $x$ of $E$, that is: the estimate error $\varepsilon(t)=\hat{x}(t)-x(t)$, converges (resp. weakly, strongly, exponentially) towards zero when $t$ goes to infinity. Then such a system $\widehat{E}$ is called an (resp. weak, strong, exponential) observer.

Let us notice that one of the most important fields of observers applications is the stabilization problem by feedback control laws: indeed in infinite dimension, since the solution of the system is generally unknown, it is of interest to get an estimate of the state from the known parameters - inputs $(u, v)$ and output $y$ - to construct

Keywords and phrases. Infinite dimensional systems, nonlinear systems, observers, regularly persistent inputs, cauchy problem, mild solution.

1 Université de Metz, INRIA Lorraine, Departement de Mathématiques, bâtiment A, Ile du Saulcy, 57045 Metz Cedex 01, France; e-mail: couchour@loria.fr

2 INSA of Rouen, Departement of Mathématiques, BP. 8, 76131 Mont-Saint-Aignan Cedex, France;

e-mail: ligarius@insa-rouen.fr 
the stabilizing control. So the observer problem finds many industrial or engineering applications in fields of robotics, mechanics, heat transfer or (bio)chemical processes (see $[1,8,9,17,19,26,27,32,35,39]$ and many other references therein and also examples in Sect. 6).

We now recall some results on linear and nonlinear systems in finite and infinite dimension, and the basic ideas allowing in our context, to construct very simple observers for a wide class of nonlinear systems.

A control system is said to be observable if for any pair of distinct initial states there is an input which gives rise to a pair of distinct outputs. For finite dimensional linear systems, any input is suitable in the definition of observability and the simpler solution of the observer problem is given by the Luenberger observer (see [12]). For nonlinear systems, even if the system is observable, one of the major difficulties for synthesis of observers, is the existence of "bad" inputs (or non universal inputs, in the sense of Sussman [33] or Sontag [34]) for which there is some pair of distinct states giving a same output function (see also Ex. 6.1 in Sect. 6). As said before, the bad input phenomenon does not exist for linear systems, but already appears on bilinear ones (that is $\mathcal{A}_{u}(t):=A$ linear, and $B(v(t), x(t)):=v(t) B_{1} x(t)$, where $B_{1}$ is linear and $v$ real-valued). From the observability point of view, bilinear systems can be treated as linear time dependent systems. And for linear time dependent systems, the Kalman's observer provides a solution. One can easily show that for linear time dependent systems that are dissipative for almost all values of $t \geq 0$, the Luenberger observer also works (see $[20,21])$.

In infinite dimension, the observer problem taking into account non universal inputs, has recently been examined in Hilbert spaces in the case of skew adjoint or more general dissipative bilinear systems (see $[22,26,38]$ or [27]), and for which the authors exhibit a particular case of the observer used in this paper. Classically, in the bilinear case in Hilbert spaces, the Gram-observability operator is usually used to give, roughly speaking, a "measure of observability" of the system. In this case, if the observation operator $C$ has a finite rank, then the Gram-observability operator is compact and cannot be coercive. But if $C$ has an infinite rank and if the Gram-observability operator is not compact, it happens that this Grammian operator is coercive. In this last case, as in finite dimension, many solutions are possible and the observation problem can be solved, for instance, by an infinite dimensional Kalman's observer (see [9]). Physically, however, only a finite number of observations makes sense, and thus the observation operator $C$ must have a finite rank. Consequently, in order to take into account the finite rank case, the Kalman's observer is not appropriate for our observation problem. That justifies (in part) our choice of a Luenberger-like observer, but the lack of coercive property will be boring: in particular, in our knowledge there is no result of existence of universal inputs in this infinite dimensional non coercive case.

This paper consists of two different things: on the one hand an abstract part namely the construction for suitable inputs $(u, v)$ of a general class of observers in an abstract nonlinear (even non bilinear) framework on reflexive Banach spaces (see Ths. 5.1 for weak observers and $\mathbf{5 . 2}$ for strong observers); and on the other hand applications of the abstract part to real systems for which we detail the existence of universal inputs or the problem of the strong convergence of the estimate error (see Sect. 6).

The required existence of universal inputs in the sense of this paper (as in [38] and [22]) is a necessary condition for the synthesis of observers. We underline that this condition implies the observability of an "asymptotic" system (deduced from the error estimate system). In particular the universal input assumption guarantees that the error estimate system does not contain any undetectable dynamic (i.e. unstable unobservable state) and so we do not need any extra stability condition (see $[29,30]$ ) on the space of undetectable states as in the classical linear case. In this paper a universal input is universal in the Susmann's sense (see [33] and [12]) with in addition a strong observability assumption. Of course, existence of universal inputs is a sharp problem and in our knowledge no theoretical result is available on this subject in infinite dimension with possibly compact outputs. In this paper this question is only tackled on examples (see Props. 6.1, 6.2, 6.3, 6.5 and Cor. 6.1).

In our general context, we had to overcome several difficulties: firstly, the nonlinearity of $B$ which, in particular, excludes the use of the Gram-observability operator; secondly, the possible compactness of the outputs; thirdly, the existence of non universal inputs (see Ex. 6.2 in Sect. 6); fourthly, the construction of an observer in reflexive Banach spaces; finally, the generality of our assumptions on systems $E$. 
We point out that in a general framework including hyperbolic and parabolic systems with possibly compact outputs, in infinite dimensional spaces it is not possible, without severe restrictions on assumptions of our system $E$, to hope for a better result than a weak observer. Such a weak convergence result (Ths. 5.1) reduces the strong convergence problem to the study of precompactness of trajectories of the system, and allows to understand why in numerical finite dimensional approximations, the estimate error converges towards zero. In the hyperbolic case for instance, the problem of strong convergence and, in particular, exponential convergence (expected in applications), requires an extra work which must take into account the specificity of the systems under consideration. Clearly, our weak convergence principle will be a first step in this direction.

Our results fully generalize, within an abstract nonlinear framework, those given in [27], [26] or similar ones in [38] or [22] for bilinear systems, and extend in several ways observer results existing in the literature (see $[9,11,18,22,26]$, and others).

This paper is organized as follows. General notations and conventions are given in Section 2 . Section 3 is devoted to some preliminary results on abstract semilinear Cauchy problems governed by time dependent families of unbounded operators. Sections 4, 5 and 6 make up the core of this article. Section 4 details assumptions on $E$. Section 5 contains the description of the considered class of observers and the statement of the abstract results while applications can be found in Section 6 . The main proofs are included in Section 7 and precede a short conclusion in Section 8. We have postponed in an appendix Section 9 numerous examples of families $\left(\mathcal{A}_{u}(t)\right)$ considered in system $E$.

\section{Conventions And notations}

Let $X$ be a reflexive Banach space. We denote by $X^{*}$ the topological dual of $X$, and the duality brackets are set by $\langle\cdot, \cdot\rangle:=\langle\cdot, \cdot\rangle_{X, X^{*}}$. The notation $\mathcal{L}(X)$ represents the set of linear bounded maps from $X$ to $X$ and $X_{\mathrm{w}}$ denotes the space $X$ endowed with the weak topology. The operator $D^{*}$ stands for the adjoint of the linear operator $D$ from $X$ to $X$.

The usual weak (resp. weak*) convergence in a Banach space $X$ is represented by the symbol " $\longrightarrow$ w" (resp. " $\stackrel{\mathrm{w}^{*}}{\longrightarrow}$ ). The set of continuous functions from $[0, T]$ to $X$, endowed with the supremum norm, is denoted by $\mathbb{C}([0, T], X)$, while $\mathbb{C}\left([0, T], X_{\mathrm{w}}\right)$ stands for the set of continuous functions from $[0, T]$ to $X_{\mathrm{w}}$ equipped with the topology of the uniform weak convergence.

Let $I_{0}$ be an arbitrary interval of $\left[0,+\infty\left[\right.\right.$. In the sequel for $p \in[1,+\infty]$ we denote by $\mathbb{L}^{p}\left(I_{0},\left(\mathbb{R}^{+}\right)^{m}\right)$ the set of $u=\left(u_{1}, \ldots, u_{m}\right) \in \mathbb{L}^{p}\left(I_{0},(\mathbb{R})^{m}\right)$ such that $u_{j} \geq 0$ a.e. on $I_{0}, j=1, \ldots, m$. As usual, we will denote $\mathbb{L}^{p}\left(I_{0}, \mathbb{R}\right)$ by $\mathbb{L}^{p}\left(I_{0}\right)$. The set of admissible controls $(u, v)$ for $E$ in $(1)$ is denoted by $\mathcal{U} \times \mathcal{V} \subset \mathbb{L}^{\infty}\left(\left[0,+\infty\left[, \mathbb{R}^{m} \times \mathbb{R}^{q}\right)\right.\right.$.

Let $u \in \mathcal{U}$, let $T>0$ and let $\tau>0$, we set by $u_{[\tau]}^{T}(\cdot)=u(\tau+\cdot)$, the $\tau$-translated input function on $[0, T]$. The set $\mathcal{U}_{T}\left(\right.$ resp. $\left.\mathcal{V}_{T}\right)$ denotes the restriction of the controls on the compact interval $[0, T]$, e.g. if $\mathcal{U}:=\mathbb{L}^{\infty}([0,+\infty[)$ then $\mathcal{U}_{T}:=\mathbb{L}^{\infty}([0, T])$. We suppose given a concept of convergence on $\mathcal{U} \times \mathcal{V}$. Then, in applications this convergence may be associated with the topology on $\mathcal{U}_{T}$ (resp. $\mathcal{V}_{T}$ ) induced by one of the following topologies on $\mathbb{L}^{\infty}\left([0, T], \mathbb{R}^{m}\right)$ (resp. $\left.\mathbb{L}^{\infty}\left([0, T], \mathbb{R}^{q}\right)\right)$ :

a) the weak (or weak* or strong) topology of $\mathbb{L}^{\infty}\left([0, T], \mathbb{R}^{m}\right)\left(\operatorname{resp} . \mathbb{L}^{\infty}\left([0, T], \mathbb{R}^{q}\right)\right)$;

b) the relative strong topology of $\mathbb{L}^{1}\left([0, T], \mathbb{R}^{m}\right)\left(\operatorname{resp} . \mathbb{L}^{1}\left([0, T], \mathbb{R}^{q}\right)\right)$ on $\mathbb{L}^{\infty}\left([0, T], \mathbb{R}^{m}\right)\left(\operatorname{resp} . \mathbb{L}^{\infty}\left([0, T], \mathbb{R}^{q}\right)\right)$. The notation $u_{n} \stackrel{\mathcal{U}_{T}}{\longrightarrow} u_{\infty}$ (resp. $\left.v_{n} \stackrel{\mathcal{V}_{T}}{\longrightarrow} v_{\infty}\right)$, means that the sequence $\left(u_{n}\right)_{n}$ (resp. $\left.\left(v_{n}\right)_{n}\right)$ converges towards $u_{\infty}$ (resp. $\left.v_{\infty}\right)$ in $\mathcal{U}_{T}\left(\right.$ resp. $\left.\mathcal{V}_{T}\right)$ for the convergence chosen.

In the sequel, we set $\Xi=\{(t, s) \in \mathbb{R} \times \mathbb{R}, 0 \leq s \leq t\}$.

\section{Preliminary Results}

We make precise here the solution notion used in this paper. We need the concept of linear evolution operators already developed in $[6,15,31]$ for instance. 
Definition 3.1. A function $\Phi$ from $\Xi$ to $\mathcal{L}(X)$ is called a linear evolution operator, if it satisfies the two following conditions:

i) for any fixed $x \in X$, the function $\Phi(\cdot, \cdot) x$ is continuous on $\Xi$;

ii) for all $(t, s),(s, r) \in \Xi$, the relations $\Phi(t, s) \circ \Phi(s, r)=\Phi(t, r)$ and $\Phi(t, t)=I$ hold. Moreover, the evolution operator is said to be contractive if for all $(t, s) \in \Xi,\|\Phi(t, s)\|_{\mathcal{L}(X)} \leq 1$.

\subsection{The Cauchy problem}

The following proposition extends some results given for instance in Prüss [31], or in Kato [25]. In particular, since the Dini derivatives are not convenient for computations involving functions defined only almost everywhere, we give an integral inequality in a space of distributions.

We introduce the following notation $[x, y]_{-}=\lim _{\lambda \uparrow 0}(\|x+\lambda y\|-\|x\|) / \lambda$, for all $x, y \in X$.

Let $C P_{F}\left(x^{0}\right)$ be the following evolution problem,

$$
C P_{F}\left(x^{0}\right)=\left\{\begin{array}{l}
\dot{x}(t)=A(t) x(t)+F(t, x(t)), \quad \text { on }[0,+\infty[ \\
x(0)=x^{0} \in X,
\end{array}\right.
$$

for which we always suppose satisfied,

(i) the family $(A(t))_{t>0}$ of densely defined operators from $X$ to $X$ is the generator of a linear contractive evolution operator $\Phi$;

(ii) the $X$-valued map $x \mapsto F(t, x)$ is continuous for a.a. $t \in[0,+\infty[$ and the application $\tau \mapsto F(\tau, \xi)$ is strongly measurable for all $\xi \in X$;

(iii) for each $t_{0} \geq 0$, there exists $\alpha \in \mathbb{L}^{1}\left(\left[0, t_{0}\right]\right)$ such that for all bounded subsets $\Omega$ of $X$ one has for a.a. $\tau \in\left[0, t_{0}\right]$

$$
\begin{gathered}
\chi(F(\tau, \Omega)) \leq \alpha(\tau) \chi(\Omega) \\
\sup _{x \in \Omega}\|F(\tau, x)\| \leq \beta_{\Omega}(\tau)
\end{gathered}
$$

where the symbol $\chi$ stands for the Hausdorff measure of non compactness and $\beta_{\Omega} \in \mathbb{L}^{1}\left(\left[0, t_{0}\right]\right)$;

(iv) there exists a non negative function $g \in \mathbb{L}_{\text {loc }}^{1}([0,+\infty[)$ such that for all $\xi \in X$ and a.a. $t \in[0,+\infty[$ we have

$$
[\xi, F(t, \xi)]_{-} \leq g(t)(\|\xi\|+1) .
$$

We have the following fundamental result on the Cauchy problem:

Proposition 3.1. Under assumptions (i-iv), the problem $C P_{F}\left(x^{0}\right)$ has at least a Duhamel's solution, i.e., there exists a continuous function $x$ on $[0,+\infty[$ satisfying the following relation,

$$
x(t)=\Phi(t, 0) x^{0}+\int_{0}^{t} \Phi(t, \tau) F(\tau, x(\tau)) \mathrm{d} \tau
$$

for $t \geq 0$. Moreover, if we consider $F(t, \xi)=f(t)$ with $f \in \mathbb{L}_{\mathrm{loc}}^{1}\left(\left[0,+\infty[, X)\right.\right.$, the unique solution $x$ of $C P_{f}\left(x^{0}\right)$ satisfies

in $\mathcal{D}^{\prime}(] 0,+\infty[)$.

$$
\frac{\mathrm{d}}{\mathrm{d} t}\|x(t)\| \leq[x(t), f(t)]_{-}
$$

The proof of Proposition 3.1 can be found in Section 7.2.

Remark 3.1. Comments on the Cauchy problem $C P_{F}\left(x^{0}\right)$.

1. If $\xi \mapsto F(t, \xi)$ is dissipative for a.a. $t \geq 0$ then $C P_{F}\left(x^{0}\right)$ has a unique global Duhamel's solution $x$ on $[0,+\infty[$. If in addition $F(t, 0)=0$ a.a. $t \geq 0$, from (4) it comes: $\|x(t)\| \leq\left\|x^{0}\right\|$, for all $t \in[0,+\infty[$.

2. We emphasize that in our semilinear time dependent framework, the inequality (4) (in its integrated form) improves (see [5]) the classical Benilan's integral inequality (for the general quasi-autonomous problem) in the 
sense that the upper bound in the right hand side of (4) is smaller than the usual upper bound $[x(t), f(t)]=$ $\lim _{\lambda \downarrow 0}(\|x(t)+\lambda f(t)\|-\|x(t)\|) / \lambda$.

\subsection{Compact evolution operator}

We will need in Theorem 5.2 the following concept:

Definition 3.2. We will say that the evolution operator $\Phi$ is compact if there exists $T_{1} \geq 0$ such that for all $h>0$ and all bounded subsets $\Omega$ of $X$ the set $\bigcup_{t \geq T_{1}} \Phi(t+h, t) \Omega$ is relatively compact in $X$.

\section{Example 3.1. Compact evolution operator}

If for all $t \geq T_{1}$ and all $h>0$ we have $\Phi(t+h, t)=K(h) \circ L(t, h)$ where $K(h)$ is a compact operator and where $\bigcup_{t \geq T_{1}} L(t, h) \Omega$ is bounded for each bounded subset $\Omega \subset X$, then $\Phi$ is compact.

Let us precise the notion of local uniform integrability.

Definition 3.3. We say that a function $f$ from $[0,+\infty[$ to $X$ is locally uniformly integrable on $[0,+\infty$ [ if $f$ is locally integrable on $[0,+\infty[$ and if we have,

$$
\limsup _{h \downarrow 0} \int_{a \geq 0}^{a+h}\|f(\tau)\| \mathrm{d} \tau=0 .
$$

We have the following result for systems generating compact evolution operators:

Lemma 3.1. Consider $C P_{f}\left(x^{0}\right)$ defined in (2) in case $F(t, \xi):=f(t)$. Assume $f$ to be locally uniformly integrable and assume the contractive linear evolution operator $\Phi$ (introduced in hypothesis (i) before Prop. 3.1) to be compact. Then the trajectory of the solution of $C P_{f}\left(x^{0}\right)$ is precompact if (and only if) it is bounded.

Proof. Let $x$ be the solution of $C P_{f}\left(x^{0}\right)$ and suppose that the trajectory $\Omega=x([0,+\infty[)$ is bounded. Since $\tau \mapsto x(\tau)-\Phi(\tau, t-h) x(t-h)$ is solution of (2) on $[t-h, t]$, with zero (instead of $\left.x^{0}\right)$ as initial value and $F(t, \xi)=f(t)$, it follows from Proposition 3.1,

$$
\|x(t)-\Phi(t, t-h) x(t-h)\| \leq \int_{t-h}^{t}\|f(\tau)\| \mathrm{d} \tau,
$$

for all $h \geq 0$ and $t \geq T_{1}+h$ ( $T_{1}$ is given in Def. 3.2). Thus from (5,6) and the precompactness of $\bigcup_{t \geq T_{1}+h} \Phi(t, t-h) \Omega$, the set $x\left(\left[T_{1}+h,+\infty[)\right.\right.$ is precompact. The claim of Lemma 3.1 is now obvious.

\section{Assumptions ON $E$}

Let $(u, v) \in \mathcal{U} \times \mathcal{V}$, and consider the nonlinear system $E$ given by (1) on the reflexive Banach space $X$. We define precisely below assumptions, on the family of unbounded linear operators $\left(\mathcal{A}_{u}(t)\right)$ (see Assumption (A)), on the nonlinear operator $B$ (see Assumption (B)) and on the observation operator $C$ (see Assumption (C)).

\subsection{Assumption (A)}

We denote by (A), the set of four following assumptions (A1-A4).

(A1) The family $\left(\mathcal{A}_{u}(t)\right)_{t>0}$ of densely defined operators from $X$ to $X$ is the generator of a linear contractive evolution operator $\Phi_{u}$.

(A2) The evolution operator satisfies the control-translation property, i.e. for all $(t, s) \in \Xi$ and all $u \in \mathcal{U}$, the set of controls $u^{*} \in \mathcal{U}$ such that $u^{*}(\tau) \equiv u_{[s]}(\tau)$ for $\tau \in[0, t-s]$ is non empty (recall $u_{[s]}(\tau)=u(s+\tau)$ ) and for any such $u^{*}$,

$$
\Phi_{u}(t, s)=\Phi_{u^{*}}(t-s, 0) .
$$


(A3) For all $\varphi \in X^{*}$ and for all bounded subsets $\Omega_{0} \subset X$, we have

$$
\lim _{h \downarrow 0} \sup _{x \in \Omega_{0}, t \geq 0}\left\langle\Phi_{u}(t+h, t) x-x, \varphi\right\rangle=0 .
$$

(A4) For all $\varphi \in X^{*}$, if $u_{n} \stackrel{\mathcal{U}_{T}}{\longrightarrow} u_{\infty}$ and $x_{n}^{0} \stackrel{\mathrm{w}}{\longrightarrow} x_{\infty}^{0}$ then for all $(t, s) \in \Xi \cap[0, T]^{2}$, we have,

$$
\left\langle\Phi_{u_{n}}(t, s) x_{n}^{0}, \varphi\right\rangle \rightarrow\left\langle\Phi_{u_{\infty}}(t, s) x_{\infty}^{0}, \varphi\right\rangle .
$$

Due to Assumption (A2), for $(t, s) \in \Xi \cap[0, T]^{2}$, there is no ambiguity here to consider $\Phi_{u}(t, s)$ with $u \in \mathcal{U}_{T}$ defined on $[0, T]$ only, and not on $[0,+\infty[$.

Assumption (A) is very general as we will see in numerous examples exhibited in Section 9.2 below.

Remark 4.1. Comments on Assumptions (A).

1. Assumption (A2) implies that if two elements $u, v \in \mathcal{U}$ coincide on $[s, t]$ then $\Phi_{u}(\tau, \sigma)=\Phi_{v}(\tau, \sigma)$ for $0 \leq s \leq \sigma \leq \tau \leq t$. The control-translation property is for instance satisfied in case $\mathcal{A}_{u}(t)$ depends on $u$ through $u(t)\left(\right.$ i.e. $\left.\mathcal{A}_{u}(t)=\mathcal{A}_{u(t)}\right)$ and $\mathcal{U}=\mathbb{L}^{\infty}([0,+\infty[)$. See also examples in Section 9.2.

2. In conditions (A3) and (A4) $X^{*}$ can be replaced equivalently by a dense subset $\Omega^{*}$ of $X^{*}$. Indeed, we have $\left\|\Phi_{u}^{*}(t+h, t)\right\|_{\mathcal{L}\left(X^{*}\right)}=\left\|\Phi_{u}(t+h, t)\right\|_{\mathcal{L}(X)} \leq 1$ and thus relations $(7,8)$ (and also (74) below) can be extended by density. In the same way, we can replace in relation (7) the set $\Omega_{0}$ by a dense subset $\Omega_{1}$ of $\Omega_{0}$.

\subsection{Assumption (B)}

In this section, we precise the assumptions on the nonlinear part of System $E$. As in Section 3.1 the symbol $\chi$ stands for the Hausdorff measure of non compactness. Let $\mathcal{C}_{T}$ be either $\mathbb{C}([0, T], X)$ or $\mathbb{C}\left([0, T], X_{\mathrm{w}}\right)$. The nonlinear operator $B$ defined from $\mathbb{R}^{q} \times X$ to $X$ satisfies the following conditions:

(B1) The operator $B$ is dissipative with respect to its second variable, i.e. for all $\xi \in \mathbb{R}^{q}$, and all $(x, z) \in X \times X$,

$$
[x-z, B(\xi, x)-B(\xi, z)]_{-} \leq 0 .
$$

(B2) For all $v \in \mathcal{V}$, the application $(\tau, x) \mapsto B(v(\tau), x)$ is a Caratheodory function on $\mathbb{R}^{+} \times X$, and there exists a constant $Q_{v}>0$, such that relations

$$
\chi(B(v(\tau), \Omega)) \leq Q_{v} \chi(\Omega) \quad \text { and } \quad \sup _{x \in \Omega}\|B(v(\tau), x)\| \leq \beta_{\Omega, v}(\tau)
$$

hold for almost all $\tau \geq 0$, all bounded subsets $\Omega$ of $X$, and some $\beta_{\Omega, v}$ locally uniformly integrable on $[0,+\infty[$ (see Def. 3.3).

(B3) The nonlinear operator $B$ is $\left(\mathcal{V}_{T} \times \mathcal{C}_{T}\right)$-integrably sequentially continuous in the following sense:

the relations $v_{n} \stackrel{\mathcal{V}_{T}}{\longrightarrow} v_{\infty}$ and $x_{n}(\cdot) \stackrel{\mathcal{C}_{T}}{\longrightarrow} x_{\infty}(\cdot)$ imply

$$
B\left(v_{n}(\cdot), x_{n}(\cdot)\right) \stackrel{\mathrm{w}}{\longrightarrow} B\left(v_{\infty}(\cdot), x_{\infty}(\cdot)\right) \text { in } \mathbb{L}^{1}([0, T], X) .
$$

\subsection{Assumption (C)}

The linear observation operator $C$ from $X$ to $Y$ is:

(C1) Bounded, with $Y$ being a finite or an infinite dimensional observation normed space. 


\subsection{Assumption (E1)}

(E1) The solution of $E$ is bounded on $[0,+\infty[$.

Remark 4.2. Comments on Assumptions (B, C) and (E1).

1. Assumptions (A) and (B) are not sufficient to ensure (see Prop. 3.1) the boundedness of the solution $x$ of $E$ on $\mathbb{R}^{+}$. Owing to Proposition 3.1 a usual sufficient condition of boundedness is, for instance, $B(\xi, 0)=0$, for all $\xi \in \mathbb{R}^{q}$. However in this paper the boundedness is not studied. On the one hand, in practice, the boundedness is often checked by suitable Lyapunov functions. On the other hand, in physical applications observers are obviously considered for bounded trajectories.

2. Assumption (B2) is automatically satisfied if for instance, for all $\xi \in \mathbb{R}^{q}$, the operator $B$ is Lipschitz w.r.t the second variable and bounded on bounded subsets of $\mathbb{R}^{+} \times X$. All the relations developed here remain valid if the relation (9) holds in a local suitable sense and for instance if $B$ is locally Lipschitz w.r.t the second variable.

3. A classical situation where (B3) holds is the following with $\mathcal{C}_{T}=\mathbb{C}\left([0, T], X_{\mathrm{w}}\right): B(v(t),)=.v(t) B_{1}($.$) with$

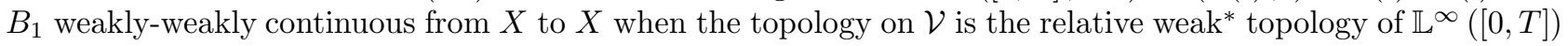
in $\mathcal{V}$. The verification of this claim is left to the reader.

4. For instance, when $v$ has positive components the dissipativity in (B1) will only be required for $\xi \in\left(\mathbb{R}^{+}\right)^{q}$.

\section{Nonlinear Asymptotic observers}

Of course, since we are concerned with the construction of observers, some asymptotic system must be observable in some sense precisely described in next Section 5.3. Let us notice that such an assumption will imply a detectability condition for the error estimate system as in the linear classical case (the unobservable dynamics are stable, see [37]).

We propose for the system $E$ given in (1), the following general asymptotic observer for all $(u, v) \in \mathcal{U} \times \mathcal{V}$,

$$
\widehat{E}=\left\{\begin{array}{l}
\dot{\hat{x}}(t)=\mathcal{A}_{u}(t) \hat{x}(t)+B(v(t), \hat{x}(t))-K(t, \widehat{y}(t)-y(t)) \\
\widehat{y}(t)=C(\hat{x}(t)) ; \quad \hat{x}(0)=\hat{x}^{0} \in X
\end{array}\right.
$$

where the nonlinear operator $K$ is precisely defined below in assumptions (O).

\subsection{Assumption (O)}

Let $M \in \mathbb{R}^{+*} \cup\{+\infty\}$ and consider the set:

$$
\Gamma_{M}=\left\{(t, \xi, \theta) ; t \geq 0,0 \leq \xi \leq M, 0 \leq \theta \leq\|C\|_{Y} \xi\right\}
$$

(O1) The map $K$ from $\mathbb{R}^{+} \times Y$ to $X$ is continuous ; for all $t \geq 0$, the map $y \mapsto K(t, y)$ is Lipschitzian, with Lipschitz constant independent of $t$, and satisfies $K(t, 0)=0$.

(O2) There exists a continuous function $h$ from $\Gamma_{\infty}$ to $\mathbb{R}^{+}$continuous on each $\Gamma_{M}, M<+\infty$, satisfying

(i) for all $t \in[0,+\infty[$, for all $x \in X$,

$$
[x,-K(t, C(x))]_{-} \leq-h\left(t,\|x\|,\|C(x)\|_{Y}\right)
$$

(ii) for each $M<+\infty$, and each sequence $\left(t_{n}, \xi_{n}, \theta_{n}\right)_{n}$ in $\Gamma_{M}$ we have

$$
\left.\begin{array}{l}
t_{n} \rightarrow+\infty \\
h\left(t_{n}, \xi_{n}, \theta_{n}\right) \rightarrow 0
\end{array}\right\} \Rightarrow\left(\theta_{n} \rightarrow 0\right)
$$

Remark 5.1. Comments on assumption (O). 
1. Whenever $K(t, 0)=0$, a.e. $t \geq 0$ (for instance $z \mapsto K(t, z)$ linear a.e. $t \geq 0$ ), Assumption (O2)-(i) implies that $x \mapsto-K(t, C(x))$ is dissipative for each $t \in[0,+\infty[$.

2. Consider a Hilbert space $X=H$, with a $\mathbb{R}$-valued output $y=C(x)=\langle x, c\rangle_{H}$, where $c \in H \backslash\{0\}$ is the observation vector ; then one can check assumptions $(\mathrm{O})$ with $K(t, C(x))=\langle x, c\rangle_{H} c$, and $h(t, \xi, \theta)=\theta^{2} / \xi$ (with $h(\cdot, 0,0)=0)$.

3. More generally, let $X=l_{p}(\phi)$ with $\phi$ an arbitrary set and $1<p<+\infty$ (recall that, $l_{p}(\phi)$ is a reflexive Banach space and any Hilbert space is isomorphic to some $\left.l_{2}(\phi)\right)$. Let $\left(e_{\varphi}\right)_{\varphi \in \phi}$ be the canonical Schauder basis of $l_{p}(\phi)$, and $x_{\varphi}$ the component of $x$ on $e_{\varphi}$. A direct computation shows that Assumption (O2) holds with $y=C(x)=x_{\varphi}, K(t, C(x))=x_{\varphi} e_{\varphi}$ and $h(t, \xi, \theta)=\theta^{p} / \xi^{p-1}$. One can observe that the inequality in (O2)-(i) becomes an equality with the function $K(t, C(x))$ chosen.

4. Obviously in the previous example with $X=l_{p}(\phi)$, Assumption (O2) remains true with any positive linear combination of $x_{\varphi} e_{\varphi}$ i.e. $y=C(x)=\left(x_{\varphi_{1}}, \cdots, x_{\varphi_{n}}\right)^{\top}$ and for all $t \geq 0, K(t, C(x))=\sum_{i}^{n} \lambda_{i} x_{\varphi_{i}} e_{\varphi_{i}}$ with $\lambda_{i}>0$ and $n \in \mathbb{N}$.

\subsection{The error equation}

The estimate error $\varepsilon(t)=\hat{x}(t)-x(t)$ satisfies the following equation:

$$
\Sigma=\left\{\begin{array}{l}
\dot{\varepsilon}(t)=\mathcal{A}_{u}(t) \varepsilon(t)+\Delta_{\hat{x}} B_{v}(t, \varepsilon(t))-K(t, C(\varepsilon(t))) \\
\varepsilon(0)=\varepsilon^{0} \in X
\end{array}\right.
$$

where $\Delta_{\hat{x}} B_{v}(t, \varepsilon):=B(v(t), \hat{x}(t))-B(v(t), \hat{x}(t)-\varepsilon)$. Let $z$ be a continuous function from $[0,+\infty[$ to $X$, and denote by $\Psi_{u, v}^{z}(t, s)$ the nonlinear evolution operator associated with $\xi \mapsto \mathcal{A}_{u}(t) \xi+\Delta_{z} B_{v}(t, \xi):=\mathcal{A}_{u}(t) \xi+$ $B(v(t), z)-B(v(t), z-\xi)$ (see Prop. 3.1). This means that $\Psi_{u, v}^{z}(t, s) \varepsilon^{0}$ is the value at $t$ of the solution of $\Sigma$ on $\left[s,+\infty\left[\right.\right.$, with $\Delta_{z}$ instead of $\Delta_{\hat{x}}$ and $\varepsilon(s)=\varepsilon^{0}$ instead of $\varepsilon(0)=\varepsilon^{0}$.

Remark 5.2. In the bilinear case, i.e. $B(v(t), x):=v(t) D x$ with $D$ bounded linear, we have simply in the error equation $\Delta_{z} B_{v}(t, \varepsilon)=v(t) D \varepsilon$ for all continuous functions $z$.

\subsection{Universal and regularly persistent inputs}

We have now to characterize the class of inputs which guarantee the observability properties of the system $E$. As in the next definition, if necessary we make more precise the notation of the system $E$ by setting $E=$ $E_{u, v}\left(x_{0}\right)$.

We adapt here the classical notions of universal and regularly persistent inputs introduced first in finite dimension by Sussmann [33] and Sontag [34] and by Gauthier et al. [22] in infinite dimension:

Definition 5.1. An input $\left(u_{\infty}, v_{\infty}\right) \in \mathcal{U}_{T} \times \mathcal{V}_{T}$ is said to be universal on $[0, T]$ if,

$$
\left(z^{0} \neq z^{1}\right) \Rightarrow\left(C \circ S_{u_{\infty}, v_{\infty}}^{T}\left(z^{0}\right) \neq C \circ S_{u_{\infty}, v_{\infty}}^{T}\left(z^{1}\right)\right)
$$

where, $S_{u_{\infty}, v_{\infty}}^{T}\left(x^{0}\right)$ denotes the restriction on $[0, T]$ of the solution of $E_{u_{\infty}, v_{\infty}}\left(x^{0}\right)$, for all $x^{0} \in X$.

Remark 5.3. Notice, as in [22], that the notion of universal controls $\left(u_{\infty}, v_{\infty}\right)$ on $[0, T]$ is a generalization of initial observability on $[0, T]$ for linear systems defined, for instance in Curtain-Pritchard [16] (p. 69).

Another equivalent definition of universal inputs can be given as follows:

Lemma 5.1. An input $\left(u_{\infty}, v_{\infty}\right) \in \mathcal{U}_{T} \times \mathcal{V}_{T}$ is universal on $[0, T]$ if and only if, for each solution $(z(t), t \in[0, T])$ of $E_{u_{\infty}, v_{\infty}}\left(z^{0}\right)$, we have the following implication, for all $\varepsilon^{0} \in X$,

$$
\left(\forall t \in[0, T], C\left(\Psi_{u_{\infty}, v_{\infty}}^{z}(t, 0) \varepsilon^{0}\right)=0\right) \Rightarrow\left(\varepsilon^{0}=0\right) .
$$


Definition 5.2. An input $(u, v) \in \mathcal{U} \times \mathcal{V}$ is said to be regularly persistent if there exist $T>0$, and a strictly increasing sequence $\left(\tau_{n}\right)_{n}$ of real positive numbers satisfying,

i) $\lim _{n} \tau_{n}=+\infty$;

ii) $T_{0}=\sup _{n}\left(\tau_{n+1}-\tau_{n}\right)<+\infty$ and the sequence $\left(u_{\left[\tau_{n}\right]}^{T_{0}}, v_{\left[\tau_{n}\right]}^{T_{0}}\right)$ is relatively compact in $\mathcal{U}_{T_{0}} \times \mathcal{V}_{T_{0}}$;

iii) $\left(u_{\left[\tau_{n}\right]}^{T}, v_{\left[\tau_{n}\right]}^{T}\right) \stackrel{\mathcal{U}_{T} \times \mathcal{V}_{T}}{\longrightarrow}\left(u_{\infty}^{T}, v_{\infty}^{T}\right)$;

iv) the control $\left(u_{\infty}^{T}, v_{\infty}^{T}\right)$ is universal on $[0, T]$ in $\mathcal{U}_{T} \times \mathcal{V}_{T}$.

Definition 5.3. An input $(u, v) \in \mathcal{U} \times \mathcal{V}$ is said to be persistent if there exist $T>0$, and a strictly increasing sequence $\left(\tau_{n}\right)_{n}$ of real positive numbers satisfying, i), iii) and iv) in Definition 5.2.

Remark 5.4. Comments on universal, persistent and regularly persistent inputs.

1. The notion of regularly persistent input developed here is a generalization of those given in [22]. Analogous inputs (which do not imply the observability since (12) is only required for distinguishable $z^{0}$ and $z^{1}$ ) have already been studied in finite dimension by Sussmann [33] and Sontag [34] or Celle et al. [12], and are extended and adapted here to an abstract infinite dimensional Banach space.

2. Thanks to Definition 5.1, a universal input separates the state points from the output, and then make the system observable on $[0, T]$. A regularly persistent input $(u, v)$ is defined on $\left(\left[0,+\infty[)^{2}\right.\right.$ and the restriction of $(u, v)$ on $([0, T])^{2}$, denoted $\left(u_{\left[\tau_{n}\right]}^{T}, v_{\left[\tau_{n}\right]}^{T}\right)$, tends to make the system observable in the same way as the universal input $\left(u_{\infty}^{T}, v_{\infty}^{T}\right)$. Roughly speaking, regularly persistent inputs are sufficiently rich to guarantee an "asymptotic estimation" of the state of the system.

3. Starting with a universal input $u$ it is not difficult to construct regularly persistent input by extending $u$ periodically. Indeed, for instance, in $\mathcal{U}=\mathbb{L}^{\infty}\left(\left[0,+\infty\left[, \mathbb{R}^{m}\right)\right.\right.$ equipped with the relative strong topology of $\mathbb{L}^{1}\left(\left[0,+\infty\left[, \mathbb{R}^{m}\right)\right.\right.$ or its strong or weak or weak*-topology, a periodic input with period $T_{0}$, which is universal on $[0, T]$ with $0<T \leq T_{0}$, is regularly persistent (see examples given in Sect. 6).

4. Definitions $5.1,5.2$ and 5.3 can be extended to the case $T=+\infty$ by replacing $[0, T]$, by $\left[0,+\infty\left[\right.\right.$ and $\mathcal{U}_{T}$ $\left(\operatorname{resp} . \mathcal{V}_{T}\right)$ by $\mathcal{U}(\operatorname{resp} . \mathcal{V})$

\subsection{The observer abstract results}

We suppose in this subsection that Assumptions (A), (B), ( $\left.\mathrm{E}_{1}\right)$ and $(\mathrm{O})$ hold.

Theorem 5.1. Let $(u, v) \in \mathcal{U} \times \mathcal{V}$ be a regularly persistent input and suppose $\mathcal{C}_{T}=\mathbb{C}\left([0, T], X_{\mathrm{w}}\right)$ in Assumption (B3). Then the estimate error $\varepsilon($.$) converges weakly to zero in X$ as $t$ goes to $+\infty$.

In the case where all the inputs are universal (see Ex. 6.2), we deduce the following corollary:

Corollary 5.1. Suppose $\mathcal{C}_{T}=\mathbb{C}\left([0, T], X_{\mathrm{w}}\right)$ in Assumption (B3). If every input $(u, v) \in \mathcal{U}_{T} \times \mathcal{V}_{T}$ is universal for some fixed $T>0$, and if the set of translated functions $\left\{\left(u_{[\tau]}^{T}, v_{[\tau]}^{T}\right) ; \tau \geq 0\right\}$ is sequentially precompact in $\mathcal{U}_{T} \times \mathcal{V}_{T}$, then the observer error $\varepsilon($.$) converges weakly to zero in X$ (when $t$ goes to $+\infty)$ for any $(u, v) \in \mathcal{U} \times \mathcal{V}$.

The next strong observer principle concerns an important class of systems. We emphasize that in this result, only $\mathcal{C}_{T}=\mathbb{C}([0, T], X)$ is required in Assumption (B3).

Theorem 5.2. Suppose $\mathcal{C}_{T}=\mathbb{C}([0, T], X)$ in Assumption (B3) and let $(u, v) \in \mathcal{U} \times \mathcal{V}$ be a persistent input. If $\Phi_{u}$ is compact then the observer error $\varepsilon($.$) converges strongly towards zero in X$ as $t$ goes to $+\infty$.

\section{Applichtions}

We give in this section various examples for the observation problem with some developments about the existence of regularly persistent inputs and about the strong convergence of the estimate error.

Example 6.1. A finite dimensional system. 
The following elementary bilinear example with $x=\left(x_{1}, x_{2}\right)^{\top} \in \mathbb{R} \times \mathbb{R}$,

$$
\left(P_{1}\right)\left\{\begin{array}{rl}
\dot{x}_{1}(t)= & -u(t)\left(x_{1}(t)+x_{2}(t)\right) \\
\dot{x}_{2}(t)= & -x_{2}(t) \\
& y(t)=x_{1}(t),
\end{array} \quad x(0)=x_{0} \in \mathbb{R}^{2}\right.
$$

with $u \in \mathcal{U}:=\left\{\mathbb{L}^{\infty}\left(\left[0,+\infty\left[; \mathbb{R}^{+}\right), 0 \leq u(t) \leq \sqrt{2}\right.\right.\right.$, for a.a $\left.t \geq 0\right\}$, is dissipative for almost all $t \geq 0$. It is easily verified that the system $\left(P_{1}\right)$ is unobservable for $u=0$. The input $u=0$ is a non universal input for the state reconstruction problem, but each non zero input in $\mathcal{U}$ is universal (see other examples in [12]). Let us notice that such a system is observable and thus does not have undetectable states.

Example 6.2. A bilinear system with/without bad inputs [22].

We examine below a particular bilinear example, in two main situations: the case $\mathbf{A}$ of the multi-output system (i.e. $\left.y(t)=\left(y_{1}(t), y_{2}(t)\right)^{\top}\right)$ and the case $\mathbf{B}$ of single output $\left(\right.$ i.e. $\left.y(t)=y_{1}(t)\right)$. In case $\mathbf{A}$, we will show that all inputs defined on any time interval are universal, and in case $\mathbf{B}$, we will exhibit regularly persistent inputs (and bad inputs which make the system unobservable). Such a situation is in agreement with the general theory developed in finite dimension by Gauthier and Kupka in [20] or in [21].

We construct below a Luenberger observer for the considered system, and we show the weak convergence of the estimate error for all inputs in the case A (Cor. 5.1) and for all regularly persistent inputs in the case B (Th. 5.1).

Let us consider, the following bilinear system on $X=\mathbb{L}^{2}(\mathbb{R}) \times \mathbb{L}^{2}(\mathbb{R})$ with the usual scalar product $\langle\cdot, \cdot\rangle_{X}=$ $\langle\cdot, \cdot\rangle_{\mathbb{L}^{2}}+\langle\cdot, \cdot\rangle_{\mathbb{L}^{2}}$, with $\phi=\left(\phi_{1}, \phi_{2}\right)^{\top}$,

$$
\begin{array}{r}
\left(P_{2}\right)\left\{\begin{array}{l}
\frac{\partial \phi_{1}(x, t)}{\partial t}=\frac{\partial \phi_{1}(x, t)}{\partial x}+v(t) \phi_{2}(x, t) \\
\frac{\partial \phi_{2}(x, t)}{\partial t}=\frac{\partial \phi_{2}(x, t)}{\partial x}-v(t) \phi_{1}(x, t)
\end{array}\right. \\
y(t)=C(\phi(\cdot, t)), \quad\left(\phi_{1}, \phi_{2}\right)(., 0)=\left(\phi_{10}, \phi_{20}\right),
\end{array}
$$

where $y(t)=\left(y_{1}(t), y_{2}(t)\right)^{\top}=C\left(\phi_{1}, \phi_{2}\right)^{\top}$ is defined by

$$
y_{1}(t)=r_{1}\left\langle\phi_{1}(\cdot, t), \mathrm{e}^{-\frac{(\cdot)^{2}}{2}}\right\rangle_{\mathbb{L}^{2}} \quad \text { and } \quad y_{2}(t)=r_{2}\left\langle\phi_{2}(\cdot, t), \mathrm{e}^{-\frac{(\cdot)^{2}}{2}}\right\rangle_{\mathbb{L}^{2}}
$$

with $r_{j} \in\{0,1\}$ : in the multi-output case (case $\mathbf{A}$ ) we have $r_{1}=r_{2}=1$ and in the single-output case (case B), we have $r_{1}=1$ and $r_{2}=0$.

The system (13) can be rewritten in the form of $E$ by means of the following notations. Let $\phi=\left(\phi_{1}, \phi_{2}\right)^{\top} \in X$ and $\mathcal{A}_{u}(t)=A_{1} \oplus A_{2}$ defined on $\operatorname{Dom}\left(\mathcal{A}_{u}(t)\right)=H^{1}(\mathbb{R}) \times H^{1}(\mathbb{R})$ with

$$
A_{1}=\left(\begin{array}{ll}
1 & 0 \\
0 & 0
\end{array}\right)\left(\begin{array}{l}
\partial / \partial x \\
\partial / \partial x
\end{array}\right), \text { and } A_{2}=\left(\begin{array}{ll}
0 & 0 \\
0 & 1
\end{array}\right)\left(\begin{array}{c}
\partial / \partial x \\
\partial / \partial x
\end{array}\right)
$$

Of course we set $B(v(t), \phi)=v(t) D \phi$ where the bounded linear skew adjoint operator $D$ is defined by

$$
D \phi(x, t)=\left(\begin{array}{cc}
0 & 1 \\
-1 & 0
\end{array}\right) \phi(x, t)
$$

We consider $\mathcal{V}=\mathbb{L}^{\infty}\left(\left[0,+\infty[)\right.\right.$ and on $\mathcal{V}_{T}$ we can choose one of the topologies given in a), b) of Section 2 .

The operator $A_{1} \bigoplus A_{2}$ is m-dissipative densely defined on $X$. So according to Example 9.2 and Remark 4.2-3, we see that Assumptions (A), (B) and (C) hold. 
In view of the Remark 5.1, we propose the following Luenberger observer for instance in the worst case $y(t)=y_{1}(t)=\left\langle\phi_{1}(\cdot, t), \mathrm{e}^{-\frac{(\cdot)^{2}}{2}}\right\rangle_{\mathbb{L}^{2}}$. Namely for all $\hat{\phi}=\left(\hat{\phi}_{1}, \hat{\phi}_{2}\right)^{\top} \in X$, let $\hat{y}_{j}(t)=\int_{-\infty}^{+\infty} \hat{\phi}_{j}(x, t) \mathrm{e}^{-\frac{x^{2}}{2}} \mathrm{~d} x$ and

$$
\left\{\begin{array}{l}
\frac{\partial \hat{\phi}_{1}(x, t)}{\partial t}=\frac{\partial \hat{\phi}_{1}(x, t)}{\partial x}+v(t) \hat{\phi}_{2}(x, t)-r_{1}\left(\int_{-\infty}^{+\infty} \hat{\phi}_{1}(x, t) \mathrm{e}^{-\frac{x^{2}}{2}} \mathrm{~d} x-y_{1}(t)\right) \mathrm{e}^{-\frac{x^{2}}{2}} \\
\frac{\partial \hat{\phi}_{2}(x, t)}{\partial t}=\frac{\partial \hat{\phi}_{2}(x, t)}{\partial x}-v(t) \hat{\phi}_{1}(x, t)-r_{2}\left(\int_{-\infty}^{+\infty} \hat{\phi}_{2}(x, t) \mathrm{e}^{-\frac{x^{2}}{2}} \mathrm{~d} x-y_{2}(t)\right) \mathrm{e}^{-\frac{x^{2}}{2}}
\end{array}\right.
$$

We can check that the solution of (13) can be written as follows

$$
\left\{\begin{array}{l}
\phi_{1}(x, t)=\cos \left[\int_{0}^{t} v(\tau) \mathrm{d} \tau\right] \phi_{10}(x+t)+\sin \left[\int_{0}^{t} v(\tau) \mathrm{d} \tau\right] \phi_{20}(x+t) \\
\phi_{2}(x, t)=-\sin \left[\int_{0}^{t} v(\tau) \mathrm{d} \tau\right] \phi_{10}(x+t)+\cos \left[\int_{0}^{t} v(\tau) \mathrm{d} \tau\right] \phi_{20}(x+t) .
\end{array}\right.
$$

\section{Case A: The multi-outputs case.}

Consider first, the system (13) with two outputs, $y(t)=\left(y_{1}(t), y_{2}(t)\right)^{\top}$ with $y_{1}$ and $y_{2}$ as in equations (15).

Proposition 6.1. Every input $v \in \mathbb{L}^{\infty}([0, \infty[)$ is universal on $[0, T]$ for each $T>0$.

Indeed, suppose for all $t \in[0, T]$, and for all $\phi_{0} \in X$, the relation $C\left(\Psi_{v}^{z}(t, 0) \phi_{0}\right)=0$ hold. That is equivalent to the following, for all $t \in[0, T]$, and for all $\phi_{0}=\left(\phi_{10}, \phi_{20}\right)^{\top} \in X$,

$$
\left\{\begin{array}{r}
\cos \left[\int_{0}^{t} v(\tau) \mathrm{d} \tau\right] \int_{-\infty}^{+\infty} \mathrm{e}^{-\frac{x^{2}}{2}} \phi_{10}(x+t) \mathrm{d} x+\sin \left[\int_{0}^{t} v(\tau) \mathrm{d} \tau\right] \int_{-\infty}^{+\infty} \mathrm{e}^{-\frac{x^{2}}{2}} \phi_{20}(x+t) \mathrm{d} x=0 \\
-\sin \left[\int_{0}^{t} v(\tau) \mathrm{d} \tau\right] \int_{-\infty}^{+\infty} \mathrm{e}^{-\frac{x^{2}}{2}} \phi_{10}(x+t) \mathrm{d} x+\cos \left[\int_{0}^{t} v(\tau) \mathrm{d} \tau\right] \int_{-\infty}^{+\infty} \mathrm{e}^{-\frac{x^{2}}{2}} \phi_{20}(x+t) \mathrm{d} x=0
\end{array}\right.
$$

or for all $t \in[0, T]$

$$
\left\{\begin{array}{l}
\int_{-\infty}^{+\infty} \mathrm{e}^{-\frac{x^{2}}{2}} \phi_{10}(x+t) \mathrm{d} x=0 \\
\int_{-\infty}^{+\infty} \mathrm{e}^{-\frac{x^{2}}{2}} \phi_{20}(x+t) \mathrm{d} x=0
\end{array}\right.
$$

Differentiating the above expression with respect to $t$, integrating by part and evaluating at $t=0$ one get successively:

$$
\left\{\begin{array}{l}
\int_{-\infty}^{+\infty} x^{n} \mathrm{e}^{-\frac{x^{2}}{2}} \phi_{10}(x) \mathrm{d} x=0 \\
\int_{-\infty}^{+\infty} x^{n} \mathrm{e}^{-\frac{x^{2}}{2}} \phi_{20}(x) \mathrm{d} x=0
\end{array} \quad \text { with } n=0,1,2, \ldots\right.
$$

Since the Hermite-like functions $\left\{x^{n} \mathrm{e}^{-\frac{x^{2}}{2}}\right\}$ form a basis in $\mathbb{L}^{2}(\mathbb{R})$, it follows that $\phi_{0}=0$, which is what we want to prove. 


\section{Case B: The single output case.}

Consider now only one output, for instance $y(t)=y_{1}(t)=\left\langle\phi_{1}(\cdot, t), \mathrm{e}^{-\frac{(\cdot)^{2}}{2}}\right\rangle_{\mathbb{L}^{2}}$. The control $v(t)=0$ is not universal on $[0, T]$, as we can see by taking two distinct initial conditions of the form $\phi_{0}=\left(0, \phi_{20}\right)^{\top}$ since thanks to (17) the two corresponding solutions must have the same output trajectory.

Thanks to Theorem 5.1, if $v$ is regularly persistent the estimate error of the proposed observer converges weakly towards zero when $t \rightarrow+\infty$.

Proposition 6.2. System (13, 14) with output $y_{1}$ admits regularly persistent inputs.

Proof. We have to build a regularly persistent input. Let $T>0$ and $v^{T} \in \mathbb{C}^{\infty}([0, T]$ ) (the space of infinitely differentiable real functions on $[0, T]$ ) satisfying,

$$
\left.\left(v^{T}\right)^{(k)}(0)=0 \forall k \in \mathbb{N}, \text { and } v^{T}(] 0, T[) \subset\right] 0, \frac{\pi}{T}[
$$

We claim that $v$ is universal on $[0, T]$. Indeed we have to prove that if

$$
\cos \left[\int_{0}^{t} v^{T}(\tau) \mathrm{d} \tau\right] \int_{-\infty}^{+\infty} \mathrm{e}^{-\frac{x^{2}}{2}} \phi_{10}(x+t) \mathrm{d} x+\sin \left[\int_{0}^{t} v^{T}(\tau) \mathrm{d} \tau\right] \int_{-\infty}^{+\infty} \mathrm{e}^{-\frac{x^{2}}{2}} \phi_{20}(x+t) \mathrm{d} x=0
$$

for all $t \geq 0$, then we have $\left(\phi_{10}, \phi_{20}\right)=(0,0)$. Remark first that the function $t \mapsto \int_{-\infty}^{+\infty} \mathrm{e}^{-\frac{x^{2}}{2}} \phi_{10}(x+t) \mathrm{d} x$ is infinitely differentiable on $\mathbb{R}^{+}$. Let $\mathcal{I}$ be the following ideal of the ring $\mathbb{C}^{\infty}([0, T])$

$$
\mathcal{I}=\left\{f \in \mathbb{C}^{\infty}([0, T]) ; f^{(k)}(0)=0 \quad \forall k \in \mathbb{N}\right\}
$$

Denoting by $g(t)$ the left hand side of $(19)$ the estimation of $g^{(k)}(t)$ modulo the ideal $\mathcal{I}$ gives the following congruence

$$
g^{(k)}(t) \equiv \int_{-\infty}^{+\infty} P_{k}(x) \mathrm{e}^{-\frac{x^{2}}{2}} \phi_{10}(x+t) \mathrm{d} x
$$

where $P_{k}$ is a (monic) polynomial function of degree $k$ for all $k \in \mathbb{N}$. Evaluating $g^{(k)}(0)$, we have by induction, with (19) and (20), the following relation

$$
\int_{-\infty}^{+\infty} x^{k} \mathrm{e}^{-\frac{x^{2}}{2}} \phi_{10}(x) \mathrm{d} x=0 \quad \forall k \in \mathbb{N}
$$

So by a previous argument it comes $\phi_{10}=0$. Then according to (18) the relation (19) becomes

$$
\int_{-\infty}^{+\infty} \mathrm{e}^{-\frac{x^{2}}{2}} \phi_{20}(x+t) \mathrm{d} x=0
$$

for all $t \geq 0$. We have already seen that $(22)$ provides $\phi_{20}=0$. So $v^{T}$ is universal on $[0, T]$. Then reproducing such a universal input $T$-periodically on $\left[0,+\infty\left[\right.\right.$, we obtain a regularly persistent input $v \in \mathbb{L}^{\infty}([0,+\infty[)$.

Example 6.3. A nonlinear hyperbolic example: the vibrating beam.

The vibrating beam has already been studied in [32] for the stabilizing control problem and in [8] for the observation problem. But the study in [8] must be resumed since strongly universal inputs (in the sense of [8] which involves a coercive Grammian) cannot exist in such a model (with compact output). In addition we 
consider a nonlinear version of the vibrating beam with a more general class of outputs. The symbol $\varphi(x, t)$ stands for the displacement of the beam and satisfies

$$
\left\{\begin{aligned}
\frac{\partial^{2} \varphi}{\partial t^{2}}(x, t) & =-\frac{\partial^{4} \varphi}{\partial x^{4}}(x, t), & & x \in] 0, L[, t \geq 0, \\
\varphi(0, t) & =\frac{\partial \varphi}{\partial x}(0, t)=0, & & t \geq 0, \\
\frac{\partial^{3} \varphi(L, t)}{\partial x^{3}} & =\frac{\partial^{2} \varphi(L, t)}{\partial t^{2}}+v_{1}(t) G_{1}\left(\frac{\partial \varphi(L, t)}{\partial t}\right), & & t \geq 0, \\
\frac{\partial^{2} \varphi}{\partial x^{2}}(L, t) & =-\frac{\partial^{3} \varphi}{\partial t^{2} \partial x}(L, t)-v_{2}(t) G_{2}\left(\frac{\partial^{2} \varphi}{\partial t \partial x}(L, t)\right), & & t \geq 0, \\
y(t) & =\left(r_{1} \frac{\partial \varphi}{\partial t}(L, t), r_{2} \frac{\partial^{2} \varphi}{\partial t \partial x}(L, t)\right)^{\top}, & & t \geq 0,
\end{aligned}\right.
$$

where for $j \in\{1,2\}, v_{j}(t)$ is an applied scalar control which acts on the free boundary of the system, $G_{j}$ a Lipschitz increasing map from $\mathbb{R}$ to $\mathbb{R}$ such that $G_{j}(0)=0$ and $r_{j} \in\{0,1\}$ with $r_{1}+r_{2} \neq 0$. The velocities $\frac{\partial \varphi}{\partial t}(L, t), \frac{\partial^{2} \varphi}{\partial t \partial x}(L, t)$ on the free boundary of the beam are set for the output $y(t)$ of the system. In addition, initial conditions are prescribed on the displacement and the velocity of the beam,

$$
\left.\varphi(x, 0)=\varphi_{0}(x) \quad \text { and } \quad \frac{\partial \varphi(x, 0)}{\partial t}=\varphi_{1}(x) \quad \text { for } x \in\right] 0, L[.
$$

The system could be set under the form $E$, more precisely

$$
\left\{\begin{array}{l}
\frac{\mathrm{d} Z}{\mathrm{~d} t}=A Z+B\left(v_{1}(t), v_{2}(t), Z\right) \\
y=C Z=\left(\left\langle c_{1}, Z\right\rangle,\left\langle c_{2}, Z\right\rangle\right)^{\top}
\end{array}\right.
$$

in the Hilbert space:

$$
H=\left\{\left(z_{1}, z_{2}, z_{3}, z_{4}\right)^{\top} \in H^{2}(0, L) \times L^{2}(0, L) \times \mathbb{R} \times \mathbb{R} ; z_{1}=\mathrm{d} z_{1} / \mathrm{d} x=0 \text { at } x=0\right\}
$$

with the inner product,

$$
\left\langle\left(z_{1}, z_{2}, z_{3}, z_{4}\right)^{\top},\left(\bar{z}_{1}, \bar{z}_{2}, \bar{z}_{3}, \bar{z}_{4}\right)^{\top}\right\rangle_{H}=\int_{0}^{L}\left(\frac{\mathrm{d}^{2} z_{1}(x)}{\mathrm{d} x^{2}} \frac{\mathrm{d}^{2} \bar{z}_{1}(x)}{\mathrm{d} x^{2}}+z_{2}(x) \bar{z}_{2}(x)\right) \mathrm{d} x+z_{3} \bar{z}_{3}+z_{4} \bar{z}_{4} .
$$

We have $c_{1}=\left(0,0, r_{1}, 0\right)^{\top}, c_{2}=\left(0,0,0, r_{2}\right)^{\top}$ and

$$
B\left(v_{1}, v_{2},\left(z_{1}, z_{2}, z_{3}, z_{4}\right)^{\top}\right)=\left(0,0,-r_{1} v_{1} G_{1}\left(z_{3}\right),-r_{2} v_{2} G_{2}\left(z_{4}\right)\right)^{\top} .
$$

Notice that $Z \longmapsto B(\sigma, \tau, Z)$ is not linear and (24) is not in the bilinear standard form.

The operator $A$ in (24) is defined by

$$
A^{\top}\left(z_{1}, z_{2}, z_{3}, z_{4}\right)^{\top}=\left(z_{2},-\frac{\mathrm{d}^{4} z_{1}}{\mathrm{~d} x^{4}}, \frac{\mathrm{d}^{3} z_{1}}{\mathrm{~d} x^{3}}(L),-\frac{\mathrm{d}^{2} z_{1}}{\mathrm{~d} x^{2}}(L)\right)^{\top}
$$

and

$$
\begin{aligned}
D(A)=\left\{\left(z_{1}, z_{2}, z_{3}, z_{4}\right) \in H^{4}(0, L) \times H^{2}(0, L) \times \mathbb{R} \times \mathbb{R} ; z_{i}(0)=\frac{\mathrm{d} z_{i}}{\mathrm{~d} x}(0)=0 \text { for } i=1,2\right. \\
\left.\quad \text { and } z_{2}(L)=z_{3}, \frac{\mathrm{d} z_{2}}{\mathrm{~d} x}(L)=z_{4}\right\} .
\end{aligned}
$$


It has been shown in [32] that $A$ is a skew-adjoint infinitesimal generator of a linear $C_{0}$ group of contraction with compact resolvent. For $v_{1} \geq 0, v_{2} \geq 0$, the map $Z \longmapsto B\left(v_{1}, v_{2}, Z\right)$ is dissipative in $H$ since $G_{j}$ is assumed increasing and all assumptions $(A),(B),\left(E_{1}\right)$ and $(O)$ are satisfied if $v_{j} \geq 0 j \in\{1,2\}$ and, for instance, if the topology on $\mathcal{V}_{T}=\mathbb{L}^{\infty}\left(\left[0,+\infty\left[, \mathbb{R}^{2}\right)\right.\right.$ is for all $T>0$, the weak* topology. Consequently in this context, from Theorem 5.1, for all regularly persistent input $v=\left(v_{1}, v_{2}\right) \geq(0,0)$, the estimate error $\varepsilon(t) \rightarrow 0$ weakly in $H$.

For such an input the proposed observer is

$$
\left\{\begin{array}{rlrl}
\frac{\partial^{2} \widehat{\varphi}(x, t)}{\partial t^{2}} & =-\frac{\partial^{4} \widehat{\varphi}(x, t)}{\partial x^{4}}, & x \in] 0, L[, t \geq 0, \\
\widehat{\varphi}(0, t) & =\frac{\partial \widehat{\varphi}(0, t)}{\partial x}=0, \\
\frac{\partial^{3} \widehat{\varphi}}{\partial x^{3}}(L, t) & =\frac{\partial^{2} \widehat{\varphi}}{\partial t^{2}}(L, t)+r_{1} v_{1}(t) G_{1}\left(\frac{\partial \widehat{\varphi}}{\partial t}(L, t)\right)-r_{3} r_{1} \frac{\partial}{\partial t}(\widehat{\varphi}-\varphi)(L, t), & t \geq 0, \\
\frac{\partial^{2} \widehat{\varphi}}{\partial x^{2}}(L, t)+\frac{\partial^{3} \widehat{\varphi}}{\partial t^{2} \partial x}(L, t) & =-r_{2} v_{2}(t) G_{2}\left(\frac{\partial^{2} \widehat{\varphi}}{\partial t \partial x}(L, t)\right)-r_{4} r_{2} \frac{\partial^{2}}{\partial t \partial x}(\widehat{\varphi}-\varphi)(L, t), & t \geq 0, \\
y(t)=\left(r_{1} \frac{\partial \widehat{\varphi}}{\partial t}(L, t), r_{2} \frac{\partial^{2}}{\partial t \partial x} \widehat{\varphi}(L, t)\right)^{\top}, & t \geq 0,
\end{array}\right.
$$

where $\varphi$ is the solution of $(23)$ and $r_{3}>0, r_{4}>0$.

Henceforth, consider for instance $L=1$. With this condition we will see below (thanks to a result in [32]) that no undetectable mode $\left(\left(z_{n}(L), z_{n}^{\prime}(L)\right)=0\right)$ appears for beam lengths $L=1$ and such a necessary condition will be sufficient (as we will see that) to insure that all inputs are universal. In particular condition $L=1$ makes the asymptotic system deduced from (25) observable. First, setting $T=+\infty$, we have:

Proposition 6.3. The null control $v \equiv(0,0)$ is universal on $[0,+\infty[$.

Proof. Our goal is to show $\left\langle c, \mathrm{e}^{t A} x^{0}\right\rangle_{H}=0$ for all $t \geq 0$ implies $x^{0}=0$, where

$$
c=(0,0,1,0)^{\top},
$$

the proof being analogous if $c=(0,0,0,1)^{\top}$. Set $x^{0} \in H$ and suppose

$$
\left\langle c, \mathrm{e}^{t A} x^{0}\right\rangle_{H}=0 \text { for all } t \geq 0
$$

Extending $\left(H,\langle,\rangle_{H}\right)$ in a complex Hilbert space $\left(\mathcal{H},\langle,\rangle_{\mathcal{H}}\right)$ in the usual sense we obtain (see [32]) a complete othonormal system of eigenfunctions of $A$, namely $\Phi_{n}, n \in \mathbb{Z}^{*}$ with eigenvalues $\lambda_{k}=i \mu_{k}^{2}$ and $\lambda_{-n}=-i \mu_{k}^{2}$ if $k \geq 1$ with multiplicity 1 , and $0<\mu_{1}<\mu_{2}<\cdots<\mu_{n}<\cdots$. Hence we have the following spectral decomposition

$$
Z(t):=\mathrm{e}^{t A} x^{0}=\sum_{n \in \mathbb{Z}^{*}} c_{n} \mathrm{e}^{\lambda_{n} t} \Phi_{n}
$$

with

$$
\Phi_{n}=\frac{\Psi_{n}}{\left\|\Psi_{n}\right\|_{\mathcal{H}}}, \text { where } \Psi_{n}:=\left(\begin{array}{l}
z_{n} \\
\lambda_{n} z_{n} \\
\lambda_{n} z_{n}(1) \\
\lambda_{n} z_{n}^{\prime}(1)
\end{array}\right) \text { and } z_{n}=z_{-n} \text { for } n \in \mathbb{Z}^{*}
$$

We will use the following result which avoids to turn to specific properties on almost periodic functions and in particular Besicovitch spaces (as for instance in [4]). We skip its proof which can be derived from standard arguments. 
Lemma 6.1. Let $\left(\gamma_{n}\right)_{n \in \mathbb{Z}^{*}}$ be a sequence of real numbers and $\left(\Phi_{n}\right)_{n \in \mathbb{Z}^{*}}$ an orthonormal hilbertian basis in the complex Hilbert space $\mathcal{H}$. Set $x^{0} \in \mathcal{H}, c_{n}=\left\langle x^{0}, \Phi_{n}\right\rangle$ and

$$
Z(t)=\sum_{n \in \mathbb{Z}^{*}} c_{n} \mathrm{e}^{i \gamma_{n} t} \Phi_{n}, t \geq 0
$$

Then for all $k \in \mathbb{Z}^{*}$, we have

$$
\lim _{T \longrightarrow+\infty} \frac{1}{T} \int_{0}^{T} Z(t) \mathrm{e}^{-i \gamma_{k} t} \mathrm{~d} t=\sum_{j \in \Lambda_{k}} c_{j} \Phi_{j}
$$

where we have set $\Lambda_{k}=\left\{j \in \mathbb{Z}^{*} ; \gamma_{j}=\gamma_{k}\right\}$.

By using Lemma 6.1 in Section 7 and the projection on the third component (26) implies

$$
c_{n} \lambda_{n} z_{n}(1)=0 \text { for all } n \in \mathbb{Z}^{*} .
$$

Consequently if $z_{n}(1) \neq 0$ for all $n \in \mathbb{Z}^{*}$, we deduce $c_{n}=0$ for all $n \in \mathbb{Z}^{*}$ (since $\lambda_{n} \neq 0$ ) and finally from (27) $Z(t)=0$ for all $t \geq 0$, that is in other words $x^{0}=0$. Thus we have to show $z_{n}(1) \neq 0$ for all $n \in \mathbb{N}^{*}$. By contradiction let some $n$ such that $z_{n}(1)=0$. Then from $A \Phi_{n}=\lambda_{n} \Phi_{n}$ (and line 3 in (23)) it follows

$$
z_{n}^{\prime \prime \prime}(1)=\lambda_{n}^{2} z_{n}(1)=0
$$

By direct computations (see [32]) we find

$$
z_{n}(x)=\alpha_{n}\left(\sin \mu_{n} x-\sinh \mu_{n} x\right)+\beta_{n}\left(\cos \mu_{n} x-\cosh \mu_{n} x\right)
$$

for all $x \in[0,1]$ and some $\alpha_{n}, \beta_{n} \in \mathbb{R}$. Then the system $z_{n}(1)=z_{n}^{\prime \prime \prime}(1)=0$ is equivalent to

$$
\left\{\begin{array}{l}
\alpha_{n}\left(\sin \mu_{n}-\sinh \mu_{n}\right)+\beta_{n}\left(\cos \mu_{n}-\cosh \mu_{n}\right)=0 \\
\alpha_{n}\left(-\cos \mu_{n}-\cosh \mu_{n} x\right)+\beta_{n}\left(\sin \mu_{n} x-\sinh \mu_{n} x\right)=0
\end{array}\right.
$$

which has non trivial solutions $\alpha_{n}, \beta_{n}$ if and only if

$$
\sin \mu_{n} \sinh \mu_{n}=0 .
$$

But it was shown in [32] that (28) has no solution when ( $L=1$ and) $\mu_{n}$ is an eingenvalue of $A$. Therefore $\alpha_{n}=\beta_{n}=0$ and $z_{n} \equiv 0$ that is $\Phi_{n} \equiv 0$, which is a contradiction.

Now we can state the following result:

Corollary 6.1. Every $v \in \mathbb{L}^{\infty}\left(\left[0,+\infty\left[,\left(\mathbb{R}^{+}\right)^{2}\right)\right.\right.$ is universal on $[0,+\infty[$.

Proof. Let $\widehat{Z}$ be a solution of $(24)$ and $Z$ be the solution of

$$
\frac{\mathrm{d} Z}{\mathrm{~d} t}=A Z+\Delta_{\widehat{Z}} B_{v}(t, Z)
$$

with initial condition $(Z(0))$, where $\Delta_{\widehat{Z}} B_{v}(t, Z)=B(v(t), \widehat{Z}(t))-B(v(t), \widehat{Z}(t)-Z(t))$ and suppose

$$
C Z \equiv(0,0) \text { on }[0,+\infty[\text {. }
$$

From the Duhamel's formula, we have

$$
C \mathrm{e}^{t A} Z(0)=C Z(t)-\int_{0}^{t} C \mathrm{e}^{(t-\tau) A} \Delta_{\widehat{Z}} B_{v}(\tau, Z) \mathrm{d} \tau
$$


Since $(29)$ means $\left\langle c_{j}, Z(\tau)\right\rangle=0$ it follows

$$
\begin{gathered}
\Delta_{\widehat{Z}} B_{v}(\tau, Z)=\left(0,0,-r_{1} v_{1}(\tau) G_{1}\left(\left\langle c_{1}, \widehat{Z}(\tau)\right\rangle\right),-r_{2} v_{2}(\tau) G_{2}\left(\left\langle c_{2}, \widehat{Z}(\tau)\right\rangle\right)\right)^{\top} \\
-\left(0,0,-r_{1} v_{1}(\tau) G_{1}\left(\left\langle c_{1},(\widehat{Z}-Z)(\tau)\right\rangle\right),-r_{2} v_{2}(\tau) G_{2}\left(\left\langle c_{2},(\widehat{Z}-Z)(\tau)\right\rangle\right)\right)^{\top} \\
=(0,0,0,0)^{\top}
\end{gathered}
$$

on $[0,+\infty[$. Then $(31)$ and $(30)$ give

$$
C \mathrm{e}^{t A} Z(0) \equiv 0
$$

for all $t \geq 0$. Now by Proposition 6.3 relation (32) implies $Z(0)=0$, which ends the proof.

Theorem 5.2 does not apply for system (23) but with a suitable assumption on $u$ we have the following strong convergence result.

Proposition 6.4. For each uniformly continuous control $v \in \mathbb{L}^{\infty}\left(\left[0,+\infty\left[,\left(\mathbb{R}^{+}\right)^{2}\right)\right.\right.$ the estimate error (relative to (23) and (25)) $\varepsilon(t) \longrightarrow 0$ strongly in $H$ when $t \longrightarrow \infty$.

Proof. The previous developpements and in particular Corollary 6.1 show that $\varepsilon(t) \longrightarrow 0$ weakly in $H$ when $t \longrightarrow \infty$. Thus we have just to prove the precompactness of the trajectory of $\varepsilon$. First we will prove that $\varepsilon($.$) is$ uniformly continuous on $\left[0,+\infty\left[\right.\right.$. Set $v(t)=\left(v_{1}(t), v_{2}(t)\right)$. The map $\varepsilon($.$) is solution of$

$$
\left\{\begin{array}{l}
\frac{\mathrm{d} \varepsilon}{\mathrm{d} t}=A \varepsilon+B(v(t), \widehat{Z})-B(v(t), \widehat{Z}-\varepsilon)-D C \varepsilon \\
\text { with } D\left(z_{3}, z_{4}\right):=\left(0,0, r_{3} z_{3}, r_{4} z_{4}\right)^{\top}
\end{array}\right.
$$

and $\widehat{Z}$ is solution of

$$
\frac{\mathrm{d} \widehat{Z}}{\mathrm{~d} t}=A \widehat{Z}+B(v(t), \widehat{Z})-D C \varepsilon .
$$

Of course in order to apply Lemma 7.5 we will regard (33) as a quasi-autonomous evolution governed by $A$ in a natural sense. Let us write $\varepsilon=\left(\varepsilon_{1}, \varepsilon_{2}, \varepsilon_{3}, \varepsilon_{4}\right)$. Owing to the dissipativity of $B$, integral inequalities (see [5]) give immediately

$$
\begin{aligned}
\frac{1}{2}\|\varepsilon(t)\|^{2} & \leq \frac{1}{2}\|\varepsilon(0)\|^{2}-\int_{0}^{t}\langle\varepsilon(\tau), D C \varepsilon(\tau)\rangle \mathrm{d} \tau \\
& \leq \frac{1}{2}\|\varepsilon(0)\|^{2}-\int_{0}^{t} \sum_{j=1}^{2} r_{j} r_{j+2} \varepsilon_{j+2}^{2}(\tau) \mathrm{d} \tau
\end{aligned}
$$

for all $t \geq 0$. Consequently, we have

$$
r_{j} \varepsilon_{j+2}(.) \in \mathbb{L}^{2}([0,+\infty[, H), j=1,2 .
$$

Let $h \geq 0$. The dissipativity of the map $-D C$ and Bénilan's integral inequalities again give

$$
\begin{aligned}
\frac{1}{2}\|\varepsilon(t+h)-\varepsilon(t)\|^{2} \leq & \frac{1}{2}\|\varepsilon(h)-\varepsilon(0)\|^{2}+\int_{0}^{t}\langle\varepsilon(\tau+h)-\varepsilon(\tau), B(v(\tau+h), \widehat{Z}(\tau+h)) \\
& -B(v(\tau+h),(\widehat{Z}-\varepsilon)(\tau+h))-(B(v(\tau), \widehat{Z}(\tau))-B(v(\tau), \widehat{Z}(\tau)-\varepsilon(\tau)))\rangle
\end{aligned}
$$


Thus, using the linearity of $\langle.,$.$\rangle relatively to its second variable and denoting by K$ a common Lipschitz constant of $G_{1}$ and $G_{2}$, from (36) we deduce

$$
\begin{aligned}
\frac{1}{2}\|\varepsilon(t+h)-\varepsilon(t)\|^{2} \leq & \frac{1}{2}\|\varepsilon(h)-\varepsilon(0)\|^{2}+K \int_{0}^{t} \sum_{j=1}^{2} r_{j}\left(v_{j}(\tau+h)\right)\left|\varepsilon_{j+2}(\tau+h)-\varepsilon_{j+2}(\tau)\right|\left|\varepsilon_{j+2}(\tau+h)\right| \mathrm{d} \tau \\
& +K \int_{0}^{t} \sum_{j=1}^{2} r_{j}\left(v_{j}(\tau)\right)\left|\varepsilon_{j+2}(\tau+h)-\varepsilon_{j+2}(\tau)\right|\left|\varepsilon_{j+2}(\tau)\right| \mathrm{d} \tau
\end{aligned}
$$

Now (35) insures

$$
\begin{aligned}
\lim _{h \downarrow 0} \sup _{t \geq 0} & \int_{0}^{t} \sum_{j=1}^{2} r_{j}\left(v_{j}(\tau+h)\right)\left|\varepsilon_{j+2}(\tau+h)-\varepsilon_{j+2}(\tau)\right|\left|\varepsilon_{j+2}(\tau+h)\right| \mathrm{d} \tau \\
& +\int_{0}^{t} \sum_{j=1}^{2} r_{j}\left(v_{j}(\tau)\right)\left|\varepsilon_{j+2}(\tau+h)-\varepsilon_{j+2}(\tau)\right|\left|\varepsilon_{j+2}(\tau)\right| \mathrm{d} \tau=0 .
\end{aligned}
$$

Finally (36) and (37) gives the required uniform continuity.

Now, in order to apply Lemma 7.5 in Section 7 it remains to prove that $f$ is uniformly continuous on $[0,+\infty[$. Here we have

$$
f(t)=B(v(t), \widehat{Z}(t))-B(v(t), \widehat{Z}(t)-\varepsilon(t))-D C \varepsilon(t) .
$$

According to the first part of this proof we have only to show that $g=f+D C \varepsilon$ is uniformly continuous on $[0,+\infty[$. This results from the following facts: (i) $B$ is weakly-strongly continuous, (ii) $\widehat{Z}$ is weakly uniformly continuous and bounded on $[0,+\infty[$ (see Lem. 7.3), (iii) $v$ and $\varepsilon$ are uniformly continuous and bounded on $[0,+\infty[$.

Remark 6.1. No usual classical tools on precompactness results for solutions of evolution equations (as the Webb's Theorem for exponentially stable semigroup or the Dafermos Slemrod's Theorem -for dissipative operator with compact resolvent- etc.) falls within the scope of such system (33). So it was necessary to define a suitable approach (see in Sect. 7, Lem. 7.5) in order to tackle this compactness result. Really Proposition 6.4 remains valid for a more general class of inputs than the bounded uniformly continuous controls but then we would need too long developpments to prove our claims (see [14]).

Example 6.4. A heat exchanger. A nonlinear parabolic example with a non compact and nonlinear perturbation.

Let $X_{1}$ a real Hilbert space. On the Hilbert space $X=X_{1}^{m}$ endowed with its usual inner product $\langle\cdot, \cdot\rangle_{X}=\langle\cdot, \cdot\rangle_{X_{1}}+\cdots+\langle\cdot, \cdot\rangle_{X_{1}}$ consider the following nonlinear system for a given $c \in X$,

$$
\left(P_{4}\right)=\left\{\begin{array}{l}
\dot{R}(t)=\mathcal{A}_{u}(t) R(t)+B(v(t), R(t)), \quad R(0)=R_{0} \in X \\
y(t)=\langle R(t), c\rangle_{X}
\end{array}\right.
$$

where the linear operator has the following decomposition $\mathcal{A}_{u}(t)=\bigoplus_{j=1}^{m}\left(A_{0 j}+u_{j}(t) A_{1 j}\right)$. The closed dissipative linear operators $A_{1 j}$ and $A_{0 j}$ must satisfy the following conditions namely Assumption (H):

(HA) For each $j=1, \ldots, m$ the operator $A_{0 j}$ is m-dissipative densely defined and self adjoint. For all $j=$ $1, \ldots, p \leq m$, we have

$$
\left(A_{1 j}\right)^{-1} \in \mathcal{L}(X) \text { and }\left\langle A_{0 j} x, x\right\rangle \leq-\left\|A_{1 j} x\right\|^{2}, \quad \forall x \in \operatorname{Dom}\left(A_{0 j}\right) .
$$

We suppose also that we have $A_{1 j} \in \mathcal{L}(X)$ for $j=p+1, \ldots, m$ if $p<m$. The control $u=\left(u_{1}, \ldots, u_{m}\right)$ belongs to $\mathcal{U}:=\mathbb{L}^{\infty}\left(\left[0,+\infty\left[,\left(\mathbb{R}^{+}\right)^{m}\right)\right.\right.$ and $\mathcal{U}_{T}$ is equipped with the weak ${ }^{*}$ topology. 
(HB) The operator $B$ is nonlinear continuous from $\mathbb{R}^{q} \times X$ to $X$ and satisfies Assumption (B) with $\mathcal{V}=$ $\mathbb{L}^{\infty}\left(\left[0,+\infty\left[,\left(\mathbb{R}^{+}\right)^{q}\right)\right.\right.$ and $\mathcal{V}_{T}$ equipped with the weak* topology.

When assumption (HA) holds, it is not difficult to check that Assumptions (A) is automatically satisfied for the operator $\mathcal{A}_{u}(\cdot)$. Indeed it suffices to verify that hypotheses of Example 9.3 are satisfied for $A_{0 j}+u_{j}(t) A_{1 j}$ for all $j=1, \ldots, m$. The non trivial point (explained below) is to show that $k_{j}(t)=\left\|A_{1 j} \circ \mathrm{e}^{t A_{0 j}}\right\|_{\mathcal{L}\left(X_{1}\right)}$ is locally integrable on $[0,+\infty[$ for $j=1, \ldots, p$.

Of course the linear operator $A_{1 j} \circ \mathrm{e}^{t A_{0 j}}$ is bounded for all $t>0$. The reader can check the weak-strong continuity of the operator $(t, x) \mapsto A_{1 j} \circ \mathrm{e}^{t A_{0 j}} x$ on $] 0,+\infty\left[\times X_{1}\right.$. So it is possible to show that the map $k_{j}$ is continuous on $] 0,+\infty\left[\right.$. One has classically in this analytic case for all $x_{j}^{0} \in X_{1}$, and $t>0, j=1, \ldots, p$,

$$
\left\|A_{1 j} \circ \mathrm{e}^{t A_{0 j}} x_{j}^{0}\right\|^{2} \leq-\left\langle A_{0 j} \mathrm{e}^{t A_{0 j}} x_{j}^{0}, \mathrm{e}^{t A_{0 j}} x_{j}^{0}\right\rangle \leq \frac{\left\|x^{0}\right\|^{2}}{t} .
$$

And (39) yields $k(t) \leq 1 / \sqrt{t}$ from what follows the required local integrability.

In Section 9.2 we have shown that when $\mathrm{e}^{t A_{0 j}}$ is compact for all $t>0$ (which is the case here) the evolution operator $\Phi_{u_{j}}$ is compact. Consequently, Assumption (B) in (HB) is only required with $\mathcal{C}_{T}=\mathbb{C}([0, T], X)$.

Such a system has been worked out for instance in [39] with $X_{1}=\mathbb{L}^{2}([0, L])$ and $X=X_{1}^{4}, m=4$, and models a counter flow heat exchanger in a general form,

$$
\mathcal{A}_{u}(t)=\left(\begin{array}{cccc}
\alpha_{1} D_{1}^{2}-u_{1}(t) D_{1} & 0 & 0 & 0 \\
0 & \alpha_{2} D_{2}^{2} & 0 & 0 \\
0 & 0 & \alpha_{3} D_{3}^{2}+u_{2}(t) D_{3} & 0 \\
0 & 0 & 0 & \alpha_{4} D_{4}^{2}
\end{array}\right)
$$

where $u_{i}(t) \geq 0$ stands for the fluid velocity of the counterflow heat exchanger, $\alpha_{i}>0$ is the heat diffusion coefficient and $D_{i}=\frac{\partial}{\partial x} \circ p_{i}$ where the $p_{i}$ for $i=1, \ldots, 4$ are the coordinate functions of $\mathbb{R}^{4}$ and the domains are given by the following formulae

$$
\begin{aligned}
& \operatorname{Dom}\left(D_{1}\right)=\left\{f \in W^{1,2}([0, L]) ; f(0)=0\right\}, \\
& \operatorname{Dom}\left(D_{1}^{2}\right)=\left\{f \in W^{2,2}([0, L]) ; f(0)=f^{\prime}(L)=0\right\}, \\
& \operatorname{Dom}\left(D_{l}^{2}\right)=\left\{f \in W^{2,2}([0, L]) ; f^{\prime}(0)=f^{\prime}(L)=0\right\} \text { for } l=2 \text { or } l=4, \\
& \operatorname{Dom}\left(D_{3}\right)=\left\{f \in W^{1,2}([0, L]) ; f(L)=0\right\}, \\
& \operatorname{Dom}\left(D_{3}^{2}\right)=\left\{f \in W^{2,2}([0, L]) ; f(L)=f^{\prime}(0)=0\right\} .
\end{aligned}
$$

Nevertheless in [39] as well as in the other publications devoted to this subject (see [26, 27, 40]) important simplifications (linearization, controls $u$ assumed to be constant or diffusion terms neglected etc.) were required.

We have $u=\left(u_{1}, 0, u_{2}, 0\right) \in \mathbb{L}^{\infty}\left(\left[0,+\infty\left[,\left(\mathbb{R}^{+}\right)^{4}\right)\right.\right.$. The verifications of Assumption $(\mathbf{H})$, for $\left(\mathcal{A}_{u}(t)\right)$ given by (40) and (41), are obtained by straightforward computations and are left to the reader.

The nonlinear operator $B$ is the matrix of the fluids heat exchange coefficients which vary nonlinearly with the fluid velocities, and $B$ satisfies our assumption (HB) in the working domain.

More precisely $B$ may be defined as follows. Let $R=\left(R_{1}, R_{2}, R_{3}, R_{4}\right)^{\top}$ and let $F_{1}, F_{3}$ be Lipschitz increasing maps on $\mathbb{R}$ with $F_{i}(0)=0, i=1,3$. Let also $h_{11}, h_{12}, h_{31}, h_{32}, h_{13}, h_{33}$ be positive real numbers, with $h_{12}+h_{31} \leq$ $2 \sqrt{h_{11}} \sqrt{h_{32}}$ then we set

$$
B(v, R)=\left(\begin{array}{c}
-h_{11} R_{1}+h_{12} R_{3}+h_{13}-v_{1} F_{1}\left(R_{1}\right) \\
0 \\
h_{31} R_{1}-h_{32} R_{3}+h_{33}-v_{2} F_{3}\left(R_{3}\right) \\
0
\end{array}\right)
$$


with $v=\left(v_{1}, v_{2}\right) \in \mathcal{V}=\mathbb{L}^{\infty}\left(\left[0,+\infty\left[,\left(\mathbb{R}^{+}\right)^{2}\right)\right.\right.$. For all $\xi \in\left(\mathbb{R}^{+}\right)^{2}$, the nonlinear operator $B(\xi,$.$) is dissipative,$ Lipschitz-continuous and non compact. We see that Assumption (B) holds with $\mathcal{C}_{T}=\mathbb{C}([0, T], X)$. The observation vector $c=\left(c_{1}, 0, c_{3}, 0\right)^{\top} \in X$ can be chosen to observe the first component at the output $x=L$, i.e. $y(t)=y_{1}(t)=\left\langle\phi_{1}(\cdot, t), c_{1}(\cdot)\right\rangle_{\mathbb{L}^{2}}=a^{-1} \int_{L-a}^{L} \phi_{1}(x, t) \mathrm{d} x$ with $a$ small enough (see [27], [26]).

A candidate observer satisfying $(\mathrm{O})$ for our system is the following, with $r>0$,

$$
\left(\widehat{P_{4}}\right)=\left\{\begin{array}{l}
\dot{\widehat{R}}(t)=\mathcal{A}_{u}(t) \widehat{R}(t)+B(v(t), \widehat{R}(t))-r\left(\langle\widehat{R}(t), c\rangle_{X}-y(t)\right) c \\
\widehat{R}(0)=\widehat{R}_{0} \in X .
\end{array}\right.
$$

Then Assumptions $(\mathrm{O})$ are satisfied and from Theorem 5.2 the estimate error converges strongly towards zero since $(u, v)$ is a persistent input.

Example 6.5. A non Hilbertian example. A heat equation.

Let $X=\mathbb{L}^{p}(\Omega)$, where $\left.p \in\right] 1,+\infty\left[\right.$ and $\Omega$ be a bounded open subset of $\mathbb{R}^{N}$ with a smooth boundary. Let $c \in \mathbb{L}^{p}(\Omega) \cap \mathbb{L}^{q}(\Omega)$ with $\frac{1}{p}+\frac{1}{q}=1$ and denote by $\langle.,$.$\rangle the duality bracket between \mathbb{L}^{p}(\Omega)$ and $\mathbb{L}^{q}(\Omega)$. Now, consider the following heat equation in $X$

$$
\left\{\begin{array}{l}
\frac{\mathrm{d} \varphi}{\mathrm{d} t}=\Delta \varphi+v(t) B(\varphi(t)) \\
\varphi(0)=\varphi^{0} \in X \\
y=\langle\varphi, c\rangle
\end{array}\right.
$$

where $\Delta$ is the Laplacian with domain $D=W_{0}^{1, p}(\Omega) \cap W^{2, p}$, the control $v$ belongs to $\mathcal{V}:=\mathbb{L}^{\infty}\left(\left[0,+\infty\left[, \mathbb{R}^{+}\right)\right.\right.$and $B$ is a nonlinear (non compact) dissipative Lipschitz continuous map from $X$ to $X$. The space $X$ is reflexive but not hilbertian for $p \neq 2$. Moreover (see [3] and [28]) the Laplacian with Dirichlet conditions $\Delta$ is m-dissipative with dense domain in $X$ and generates a compact semigroup in $X$. Thus assumptions (A) and (B) are satisfied with $\mathcal{C}_{T}=\mathbb{C}([0, T], X)$ for this parabolic system. A candidate observer satisfying $(\mathrm{O})$ is

$$
\left\{\begin{array}{l}
\frac{\mathrm{d} \widehat{\varphi}}{\mathrm{d} t}=\Delta \widehat{\varphi}+v(t) B(\widehat{\varphi}(t))-r\langle\widehat{\varphi}-\varphi, c\rangle c \\
\widehat{\varphi}(0)=\widehat{\varphi^{0}} \in X \\
\widehat{y}=\langle\widehat{\varphi}, c\rangle
\end{array}\right.
$$

According to Theorem 5.2, for each persistent input the error estimate $\varepsilon$ converges strongly to zero.

It is well-known (see for instance [10]) that $\Delta$ has eigenfunctions $\varphi_{n}$ in $X$ with respective negative eigenvalues $\lambda_{n}$ such that $\left(\varphi_{n}\right)_{n \in \mathbb{N}}$ form a Schauder basis of $X$ and $\lambda_{n} \downarrow-\infty$.

Suppose that there is $\alpha>0$ such that we have

$$
\sum_{n=0}^{+\infty} \mathrm{e}^{\lambda_{n} \alpha}<+\infty
$$

In particular such a technical assumption is satisfied if there exists $k \geq 2$ such that

$$
\sum_{n=0}^{+\infty} \frac{1}{\left|\lambda_{n}\right|^{k}}<+\infty
$$

which is a usual situation. 
Proposition 6.5. If (43) holds and if there is no undetectable eigenfunction then the null input is universal on $[0, T]$ for all $T>\alpha$.

Proof. Set $x(t)=\mathrm{e}^{t \Delta} x^{0}=\sum_{n=0}^{+\infty} \mathrm{e}^{\lambda_{n} t} x_{n}^{0}$ and suppose

$$
\sum_{n=0}^{+\infty} \mathrm{e}^{\lambda_{n} t}\left\langle x_{n}^{0}, c\right\rangle=0 \text { for all } t \in[0, T] .
$$

We have to prove $x^{0}=0$. Put $\alpha_{n}:=\left\langle x_{n}^{0}, c\right\rangle$.

Introduce the complex function

$$
H(z)=\sum_{n=0}^{+\infty} \mathrm{e}^{\lambda_{n} z} \alpha_{n}, z \in \mathbb{C} .
$$

Then by setting $M:=\sup _{n}\left|\alpha_{n}\right|<+\infty$, if $\operatorname{Re}(z)>\alpha$, it follows

$$
\left|\alpha_{n} \mathrm{e}^{\lambda_{n} z}\right|=\left|\alpha_{n}\right| \mathrm{e}^{\lambda_{n} \operatorname{Re}(z)} \leq\left|\alpha_{n}\right| \mathrm{e}^{\lambda_{n} \alpha} \mathrm{e}^{\lambda_{n}(\operatorname{Re}(z)-\alpha)} \leq M \mathrm{e}^{\lambda_{n} \alpha}
$$

Therefore from (43) and classical results on normal sequences of holomorphic functions, $H$ is holomorphic on the open complex half plane $\{z ; \operatorname{Re}(z)>\alpha\}$. But according to (44) the zeros of $H$ are not isolated and thus $H$ must be identically zero in this halph plane. In particular we have

$$
\sum_{n=0}^{+\infty} \mathrm{e}^{\lambda_{n} t} \alpha_{n}=0 \text { for all real numbers } t>\alpha .
$$

For all $n_{0} \in \mathbb{N}$, introduce the set $\Lambda_{n_{0}}:=\left\{n ; \lambda_{n}=\lambda_{n_{0}}\right\}$. By induction (rearranging (45) and letting $t \longrightarrow+\infty$ ) it follows

$$
\sum_{n \in \Lambda_{n_{0}}} \alpha_{n}=0
$$

for all $n_{0}$. The latter means that $\sum_{n \in \Lambda_{n_{0}}} \varphi_{n}^{0}$ is the null function (otherwise it is an undetectable eigenfunction what is impossible in view of assumptions of Prop. 6.5). Finally we have $\varphi_{n}^{0}=0$ for all $n$ which ends the proof.

\section{MAIN PROOFS}

\subsection{Proofs of the observer abstract results}

Proof of Theorem 5.1. Let us start with some preliminary lemmas.

Lemma 7.1 (Boundedness). Let $(u, v) \in \mathcal{U} \times \mathcal{V}$, and consider $x, \hat{x}$ and $\varepsilon$ respectively the solutions of $E, \widehat{E}$, and $\Sigma$ then for all $t \in\left[0,+\infty\left[\right.\right.$. Set $\|x\|_{\infty}=\sup _{t>0}\|x(t)\|$, then the following estimations hold,

$$
\begin{gathered}
\|\varepsilon(t)\| \leq\left\|\varepsilon^{0}\right\| \quad \text { and } \\
\|\hat{x}(t)\| \leq\|x\|_{\infty}+\left\|\varepsilon^{0}\right\| .
\end{gathered}
$$

This above lemma is proved directly in the next one:

Lemma 7.2. For all $(u, v) \in \mathcal{U} \times \mathcal{V} \subset\left[\mathbb{L}^{\infty}\left([0,+\infty[)]^{2}\right.\right.$, for all $T>0$, and for all $\varepsilon^{0} \in X$, we have:

$$
\lim _{t \rightarrow+\infty} \int_{0}^{T} h\left(t+\tau,\|\varepsilon(t+\tau)\|,\|C(\varepsilon(t+\tau))\|_{Y}\right) \mathrm{d} \tau=0
$$


and therefore if $\left(t_{n}\right) \uparrow+\infty$, there exists a subsequence $\left(t_{n_{k}}\right)$ such that

$$
C\left(\varepsilon\left(t_{n_{k}}+\tau\right)\right) \underset{k \rightarrow+\infty}{\longrightarrow} 0 \text { for almost all } \tau \in[0, T]
$$

Proof of Lemmas 7.1 and 7.2. From the Proposition 3.1, and the dissipativity of $z \mapsto B(v(\tau), \hat{x}(\tau))-$ $B(v(\tau), \hat{x}(\tau)-z)$ for given $\tau \in \mathbb{R}^{+}$, we obtain immediately the following inequality for $0 \leq s \leq t \leq T$,

$$
\begin{aligned}
\|\varepsilon(t)\|-\|\varepsilon(s)\| & \leq \int_{s}^{t}[\varepsilon(\tau),-K(\tau, C(\varepsilon(\tau)))]_{-} \mathrm{d} \tau \\
& \leq-\int_{s}^{t} h\left(\tau,\|\varepsilon(\tau)\|,\|C(\varepsilon(\tau))\|_{Y}\right) \mathrm{d} \tau .
\end{aligned}
$$

Then, it follows that $\|\varepsilon(\cdot)\|$ decreases on $\mathbb{R}^{+}$and $\|\varepsilon(t)\| \leq\left\|\varepsilon^{0}\right\|$. Hence $\|\hat{x}(t)\| \leq\|x(t)\|+\|\varepsilon(t)\| \leq\|x\|_{\infty}+\left\|\varepsilon^{0}\right\|$ for all $t \in[0,+\infty[$. That ends the proof of the Lemma 7.1 .

Now the second inequality of (47) implies

$$
\int_{0}^{+\infty} h\left(\tau,\|\varepsilon(\tau)\|,\|C(\varepsilon(\tau))\|_{Y}\right) \mathrm{d} \tau \leq\left\|\varepsilon^{0}\right\|
$$

Therefore, since $h$ being nonnegative, we obtain for all $T>0$,

$$
\lim _{t \rightarrow+\infty} \int_{t}^{t+T} h\left(\tau,\|\varepsilon(\tau)\|,\|C(\varepsilon(\tau))\|_{Y}\right) \mathrm{d} \tau=0
$$

which gives the first result of Lemma 7.2.

Let $\left(t_{n}\right) \uparrow+\infty$ be a sequence in $\mathbb{R}^{+}$. By the integral relation (48), the sequence of functions $\tau \mapsto$ $h\left(t_{n}+\tau,\left\|\varepsilon\left(t_{n}+\tau\right)\right\|,\left\|C\left(\varepsilon\left(t_{n}+\tau\right)\right)\right\|_{Y}\right)$ converges towards zero in $\mathbb{L}^{1}\left([0, T] ; \mathbb{R}^{+}\right)$. Consequently, there is a subsequence $\left(t_{n_{k}}\right)$ such that $h\left(t_{n_{k}}+\tau,\left\|\varepsilon\left(t_{n_{k}}+\tau\right)\right\|,\left\|C\left(\varepsilon\left(t_{n_{k}}+\tau\right)\right)\right\|_{Y}\right)$ converges towards zero for almost all $\tau \in[0, T]$. Using now the property (O2)-(ii) and Lemma 7.1, there exists a subsequence $\left(t_{n_{k}}\right)$ such that,

$$
C\left(\varepsilon\left(t_{n_{k}}+\tau\right)\right) \underset{k \rightarrow+\infty}{\longrightarrow} 0
$$

for almost all $\tau \in[0, T]$. That ends the proof of Lemma 7.2.

Lemma 7.3. Let $y$ be the Duhamel's solution of $C P_{f}\left(x^{0}\right)$ given in (2) where $f$ is a locally uniformly integrable on $\left[0,+\infty\left[\right.\right.$ (see Def. 3.3). Suppose $y$ is bounded and (A3) holds for $(A(t))$ with $\Phi$ instead of $\Phi_{u}$. Then $y$ is weakly uniformly continuous on $[0,+\infty[$. Moreover, if the trajectory $y([0,+\infty[)$ is precompact in $X$ then $y$ is strongly uniformly continuous.

Corollary 7.1. The functions $x, \hat{x}, \varepsilon$ (solution of resp. E, $\widehat{E}, \Sigma)$, are weakly uniformly continuous on $[0,+\infty[$.

Corollary 7.2. For all $\tau \in[0,+\infty[$, we have $C(\varepsilon(t+\tau)) \underset{t \rightarrow+\infty}{\stackrel{\mathrm{w}}{\longrightarrow}} 0$.

Remark 7.1. If $C$ is compact, $C(\varepsilon(t+\tau))$ converges strongly towards zero as $t$ goes to infinity.

Proof of Lemma 7.3 and its corollaries.

Step a) Proof of Lemma 7.3. Applying Proposition 3.1, By the Duhamel's formula, we can write for $t \geq 0$ and $h \geq 0$,

$$
y(t+h)-y(t)=(\Phi(t+h, t) y(t)-y(t))+\int_{t}^{t+h} \Phi(t+h, s) f(s) \mathrm{d} s .
$$


Since we have $\left\|\int_{t}^{t+h} \Phi(t+h, s) f(s) \mathrm{d} s\right\| \leq \int_{t}^{t+h}\|f(\tau)\| \mathrm{d} \tau$ the integrability assumption on $f$ (see (5)), the boundedness of $y$ on $[0,+\infty[$ and Assumption (A3) insure the required weak uniform continuity. Consequently, if the trajectory of $y$ is assumed to be precompact, we obtain the strong uniform continuity of $y$.

Step b) Proof of Corollary 7.1. According to (B2) and Lemma 7.1 we see easily that the assumptions of Lemma 7.3 are satisfied for our systems $E, \widehat{E}$ and $\Sigma$. So Corollary 7.1 is obvious.

Step c) Proof of Corollary 7.2. Now, let $T>0$ and let $\left(t_{n}\right)$ be a sequence of positive real with $\lim t_{n}=+\infty$, then the claim of Lemma 7.2 implies that there exists a subsequence $\left(t_{n_{k}}\right)$ such that

$$
\lim _{k} C\left(\varepsilon\left(t_{n_{k}}+\tau\right)\right)=0 \quad \text { for almost all } \tau \in[0, T] \text {. }
$$

The previous weak uniform continuity shown in above step a) and the linearity of $C$ give finally:

$$
C\left(\varepsilon\left(t_{n_{k}}+\tau\right)\right) \underset{k \rightarrow+\infty}{\stackrel{\mathrm{w}}{\longrightarrow}} 0, \quad \text { for all } \tau \in[0, T] .
$$

Due to the arbitrary choice of the sequence $\left(t_{n}\right)_{n}$ going to infinity we see that for each fixed $\tau \in[0,+\infty[$, it cannot exist any sequence $\left(t_{n}\right)_{n}$ such that $\left(C\left(\varepsilon\left(t_{n_{k}}+\tau\right)\right)\right)_{n}$ does not converge weakly towards zero. Therefore we have,

$$
C(\varepsilon(t+\tau)) \underset{t \rightarrow+\infty}{\stackrel{\mathrm{w}}{\longrightarrow}} 0, \quad \text { for all } \tau \in[0,+\infty[
$$

and the proof is complete.

Now we are in position to set the central lemma of the proof (which can be writing also for $T=+\infty$ provided that we replace $[0, T]$ by $[0,+\infty[)$.

Lemma 7.4. Let $\left(\tau_{n}\right)_{n \in \mathbb{N}}$ be a strictly increasing sequence in $\mathbb{R}^{+}$. Let $T>0$ be such that $\left(u_{\left[\tau_{n}\right]}^{T}, v_{\left[\tau_{n}\right]}^{T}\right) \stackrel{\mathcal{U}_{T} \times \mathcal{V}_{T}}{\longrightarrow}$ $\left(u_{\infty}, v_{\infty}\right)$, then there exists a strictly increasing sequence of positive integers $\left(n_{k}\right)_{k}$ satisfying,

i) $\hat{x}_{\left[\tau_{n_{k}}\right]}^{T}$ is weakly pointwise convergent towards some $\hat{x}_{\infty}$ on $[0, T]$;

ii) $\varepsilon_{\left[\tau_{n_{k}}\right]}^{T}$ is weakly pointwise convergent towards some $\varepsilon_{\infty}$ on $[0, T]$;

iii) $\varepsilon_{\infty}(t)=\Psi_{u_{\infty}, v_{\infty}}^{\hat{x}_{\infty}}(t, 0) \varepsilon_{\infty}(0)$;

iv) the function $\hat{x}_{\infty}$ is solution of $E_{u_{\infty}, v_{\infty}}$;

v) $\forall t \in[0, T], C\left(\Psi_{u_{\infty}, v_{\infty}}^{\hat{x}_{\infty}}(t, 0) \varepsilon_{\infty}(0)\right)=0$.

Proof. In view of Lemma 7.1 , sequences $\left(\hat{x}_{\left[\tau_{n}\right]}^{T}\right)_{n}$ and $\left(\varepsilon_{\left[\tau_{n}\right]}^{T}\right)_{n}$ are uniformly bounded in $\mathbb{C}([0, T] ; X)$.

Then a weak version of Ascoli-Arzela theorem (see Vrabie in [36], Th. 1.3.2, p. 10) guarantees the relative weak compactness of $\left(\hat{x}_{\left[\tau_{n}\right]}^{T}\right)_{n}$ and $\left(\varepsilon_{\left[\tau_{n}\right]}^{T}\right)_{n}$ in $\mathbb{C}\left([0, T], X_{\mathrm{w}}\right)$ since bounded sets of $X$ are weakly relatively compact and since the sequences $\left(\hat{x}_{\left[\tau_{n}\right]}^{T}\right)_{n}$ and $\left(\varepsilon_{\left[\tau_{n}\right]}^{T}\right)_{n}$ are weakly uniformly continuous by Lemma 7.3. Therefore, there exists a suitable increasing sequence of positive integers $\left(n_{k}\right)_{k}$ satisfying relations i) and ii) of Lemma 7.4.

For the sake of simplicity, let us write $\hat{x}_{k}, \varepsilon_{k}, u_{k}, v_{k}$ or $K_{k}$ instead of respectively $\hat{x}_{\left[\tau_{n_{k}}\right]}^{T}, \varepsilon_{\left[\tau_{n_{k}}\right]}^{T}, u_{\left[\tau_{n_{k}}\right]}^{T}, v_{\left[\tau_{n_{k}}\right]}^{T}$ or $K_{\left[\tau_{n_{k}}\right]}^{T}$. For all $\varphi \in X^{*}$, the Duhamel's relation and assumption (A2) give for all $t \in[0, T]$,

$$
\begin{aligned}
\left\langle\varepsilon_{k}(t), \varphi\right\rangle= & \left\langle\Phi_{u_{k}}(t, 0) \varepsilon_{k}(0), \varphi\right\rangle \\
& +\int_{0}^{t}\left\langle\Phi_{u_{k}}(t, s)\left\{B\left(v_{k}(s), \hat{x}_{k}(s)\right)-B\left(v_{k}(s), \hat{x}_{k}(s)-\varepsilon_{k}(s)\right)\right\}, \varphi\right\rangle \mathrm{d} s \\
& -\int_{0}^{t}\left\langle\Phi_{u_{k}}(t, s) K_{k}\left(s, C\left(\varepsilon_{k}(s)\right)\right), \varphi\right\rangle \mathrm{d} s .
\end{aligned}
$$


By virtue of Lemma 7.2, assumption (O1) and the Lebesgue dominated convergence, it comes

$$
\int_{0}^{t}\left\langle\Phi_{u_{k}}(t, s) K_{k}\left(s, C\left(\varepsilon_{k}(s)\right)\right), \varphi\right\rangle \mathrm{d} s \underset{k \rightarrow+\infty}{\longrightarrow} 0 .
$$

Now according to assumption (A4) we have,

$$
\left\langle\Phi_{u_{k}}(t, 0) \varepsilon_{k}(0), \varphi\right\rangle \underset{k \rightarrow+\infty}{\longrightarrow}\left\langle\Phi_{u_{\infty}}(t, 0) \varepsilon_{\infty}(0), \varphi\right\rangle .
$$

From (A4') and the Lebesgue dominated convergence theorem the sequence $\left(\Phi_{u_{k}}^{*}(., s) \varphi\right)_{k}$ converges (strongly) in $\mathbb{L}^{1}\left([0, T], X^{*}\right)$ towards $\Phi_{u_{\infty}}^{*}(t, s) \varphi$. Therefore both conditions (A4') and (B3) together provide

$$
\begin{aligned}
\int_{0}^{t}\left\langle B\left(v_{k}(s), \hat{x}_{k}(s)\right)-\right. & \left.B\left(v_{k}(s), \hat{x}_{k}(s)-\varepsilon_{k}(s)\right), \Phi_{u_{k}}^{*}(t, s) \varphi\right\rangle \mathrm{d} s \\
& \underset{k \rightarrow+\infty}{\longrightarrow} \int_{0}^{t}\left\langle B\left(v_{\infty}(s), \hat{x}_{\infty}(s)\right)-B\left(v_{\infty}(s), \hat{x}_{\infty}(s)-\varepsilon_{\infty}(s)\right), \Phi_{u_{\infty}}^{*}(t, s) \varphi\right\rangle \mathrm{d} s
\end{aligned}
$$

and Consequently, passing to the limit in relation (51), we conclude for all $t \in[0, T]$ and for all $\varphi \in X^{*}$,

$$
\begin{aligned}
{[c] l\left\langle\varepsilon_{\infty}(t), \varphi\right\rangle=} & \left\langle\Phi_{u_{\infty}}(t, 0) \varepsilon_{\infty}(0), \varphi\right\rangle \\
& +\int_{0}^{t}\left\langle\Phi_{u_{\infty}}(t, s)\left\{B\left(v_{\infty}(s), \hat{x}_{\infty}(s)\right)-B\left(v_{\infty}(s), \hat{x}_{\infty}(s)-\varepsilon_{\infty}(s)\right)\right\}, \varphi\right\rangle \mathrm{d} s,
\end{aligned}
$$

and finally,

$$
\forall t \in[0, T], \varepsilon_{\infty}(t)=\Psi_{u_{\infty}, v_{\infty}}^{\hat{x}_{\infty}}(t, 0) \varepsilon_{\infty}(0) .
$$

In the same way, owing to Lemma $\mathbf{7 . 2}$ for all $\varphi \in X^{*}$, and $t \in[0, T]$, we obtain easily

$$
\left\langle\hat{x}_{\infty}(t), \varphi\right\rangle=\left\langle\Phi_{u_{\infty}}(t, 0) \hat{x}_{\infty}(0), \varphi\right\rangle+\int_{0}^{t}\left\langle\Phi_{u_{\infty}}(t, s) B\left(v_{\infty}(s), \hat{x}_{\infty}(s)\right), \varphi\right\rangle \mathrm{d} s,
$$

for all $\varphi \in X^{*}$, and $t \in[0, T]$, and

$$
\hat{x}_{\infty}(t)=\Phi_{u_{\infty}}(t, 0) \hat{x}_{\infty}(0)+\int_{0}^{t} \Phi_{u_{\infty}}(t, s) B\left(v_{\infty}(s), \hat{x}_{\infty}(s)\right) \mathrm{d} s
$$

for all $t \in[0, T]$. In other words $\hat{x}_{\infty}$ is solution of $E_{u_{\infty}, v_{\infty}}$. The strong continuity of $\varepsilon_{\infty}$ and $\hat{x}_{\infty}$ which is not obvious from their definitions follows from relations (53) and (55). Thus we have shown iii) and iv).

It just remains to prove the last assertion-v) of Lemma 7.4, that is, for all $t \in[0, T], C\left(\varepsilon_{\infty}(t)\right)=0$. But this relation is clear from relation (54), Corollary 7.2 and since the bounded operator $C$ is sequentially weakly-weakly continuous (from $X$ to $Y$ ).

\section{End of proof of Theorem 5.1:}

Step a) We start with a sequence $\left(\tau_{n}\right)_{n} \uparrow+\infty$ and some $T>0$ required in the definition of regularly persistent input. In particular $\left(\tau_{n+1}-\tau_{n}\right)_{n}$ is bounded and $\left(u_{\left[\tau_{n}\right]}^{T}, v_{\left[\tau_{n}\right]}^{T}\right)_{n}$ is convergent in $\mathcal{U}_{T} \times \mathcal{V}_{T}$ towards an universal input $\left(u_{\infty}, v_{\infty}\right)$ on $[0, T]$. Then applying Lemma $\left.7.4-\mathrm{v}\right)$, and the definition of an universal input we see that each weak cluster value of $\left(\varepsilon\left(\tau_{n}\right)\right)_{n}$ in $X$ is zero. Therefore, $\varepsilon\left(t+\tau_{n}\right) \underset{n \rightarrow+\infty}{\stackrel{\mathrm{w}}{\rightarrow}} 0$ for all $t \in[0, T]$.

Step b) Finally let $\left(r_{n}\right)_{n}$ be an arbitrary sequence such that $\lim r_{n}=+\infty$. We have to prove

$$
\varepsilon\left(r_{n}\right) \underset{n \rightarrow+\infty}{\stackrel{\mathrm{w}}{\rightarrow}} 0 .
$$


In this purpose (eventually by extracting a subsequence of $\left.\left(r_{n}\right)_{n}\right)$, let us remark that we can find a sequence $\left(t_{n}\right)_{n}$ and a subsequence $\left(\tau_{q_{n}}\right)_{n}$ of $\left(\tau_{n}\right)$ satisfying

$$
\left\{\begin{aligned}
r_{n} & =\tau_{q_{n}}+t_{n}, \quad 0 \leq t_{n} \leq T_{0} \\
T_{0} & =\sup _{n}\left(\tau_{n+1}-\tau_{n}\right)<+\infty \\
\lim _{n} t_{n} & =t_{\infty} \in\left[0, T_{0}\right] .
\end{aligned}\right.
$$

Thanks to the property ii) of Definition 5.2, denote by $\left(u_{\infty}^{T_{0}}, v_{\infty}^{T_{0}}\right)$ a cluster value of $\left(\left(u_{\left[\tau_{q_{n}}\right]}^{T_{0}}\right)_{n},\left(u_{\left[\tau_{q_{n}}\right]}^{T_{0}}\right)\right)_{n}$ in $\mathcal{U}_{T_{0}} \times \mathcal{V}_{T_{0}}$, and denote respectively by $\varepsilon_{\infty}^{T_{0}}$ and $\hat{x}_{\infty}^{T_{0}}$ weak cluster values on $\left[0, T_{0}\right]$ of $\varepsilon_{\left[\tau_{q_{n}}\right]}^{T_{0}}$ and $\hat{x}_{\left[\tau_{q_{n}}\right]}^{T_{0}}$. Then Lemma 7.4-iii) with $T_{0}$ in place of $T$, yields

$$
\varepsilon_{\infty}^{T_{0}}(t)=\Psi_{\substack{x_{\infty}, v_{\infty} \\ \hat{x}_{0}}}^{T_{0}^{T_{0}}}(t, 0) \varepsilon_{\infty}^{T_{0}}(0) \quad \text { for all } t \in\left[0, T_{0}\right] .
$$

But according to Assumption (A2) we have (56) with $T_{1}=\inf \left(T_{0}, T\right)$ in place of $T_{0}$. Then in view of step a), it follows $\varepsilon_{\infty}^{T_{0}}(0)=$ weak- $\lim \varepsilon\left(\tau_{q_{n}}\right)=0$. Therefore, relation (56) becomes

$$
\varepsilon_{\infty}^{T_{0}}(t)=0 \quad \forall t \in\left[0, T_{0}\right] .
$$

Since $\varepsilon$ is weakly uniformly continuous on $\mathbb{R}^{+}$(see Lem. 7.3), the following relations holds:

$$
\varepsilon\left(r_{n}\right)-\varepsilon\left(\tau_{q_{n}}+t_{\infty}\right)=\varepsilon\left(\tau_{q_{n}}+t_{n}\right)-\varepsilon\left(\tau_{q_{n}}+t_{\infty}\right) \underset{n \rightarrow+\infty}{\stackrel{\mathrm{w}}{\rightarrow}} 0 .
$$

But since $t_{\infty} \in\left[0, T_{0}\right]$, the definition of $\varepsilon_{\infty}^{T_{0}}$ implies

$$
\varepsilon\left(\tau_{q_{n}}+t_{\infty}\right) \underset{n \rightarrow+\infty}{\stackrel{\mathrm{w}}{\longrightarrow}} \varepsilon_{\infty}^{T_{0}}\left(t_{\infty}\right)
$$

and (57) provides now $\varepsilon_{\infty}^{T_{0}}\left(t_{\infty}\right)=0$. Then, from this last equality and (58) it follows

$$
\varepsilon\left(r_{n}\right) \underset{n \rightarrow+\infty}{\stackrel{\mathrm{w}}{\rightarrow}} 0
$$

which ends the proof of Theorem 5.1.

Proof of Corollary 5.1. Let $\left(t_{n}\right)_{n}$ be a sequence in $\mathbb{R}^{+}$satisfying $\lim _{n} t_{n}=+\infty$. Let $\varepsilon^{\infty}$ be a weak cluster value of $\left(\varepsilon\left(t_{n}\right)\right)_{n}$. We want to prove $\varepsilon^{\infty}=0$.

In this goal, let us write $\varepsilon^{\infty}=$ weak-lim $\varepsilon\left(t_{n_{q}}\right)$. By Lemma 7.4, there exists a subsequence $\left(n_{q_{k}}\right)_{k}$ realizing,

$$
\forall t \in[0, T] \quad C\left(\Psi_{u_{\infty}, v_{\infty}}^{\hat{x}_{\infty}}(t, 0) \varepsilon_{\infty}(0)\right)=0
$$

with,

$$
\begin{aligned}
& \varepsilon_{\infty}=\text { weak- } \lim _{k} \varepsilon_{\left[t_{q_{k}}\right]}^{T} \\
& \widehat{x}_{\infty}=\text { weak- } \lim _{k} \widehat{x}_{\left[t_{q_{k}}\right]}^{T} \\
& u_{\infty}=\lim _{k} u_{\left[t_{q_{k}}\right]}^{T} \text { in } \mathcal{U}_{T} \text {. } \\
& v_{\infty}=\lim _{k} v_{\left[t_{q_{k}}\right]}^{T} \text { in } \mathcal{V}_{T} \text {. }
\end{aligned}
$$

Since by hypothesis, $\left(u_{\infty}, v_{\infty}\right)$ is universal on $[0, T]$ the relation $(59)$ involves $\varepsilon_{\infty}(0)=0$. Since by definition, $\varepsilon_{\infty}(0)=\varepsilon^{\infty}$, we obtain the required equality: $\varepsilon^{\infty}=0$. 
Proof of Theorem 5.2. We have to prove that, for all sequences $\left(t_{k}\right)_{k} \uparrow+\infty$ in $\mathbb{R}^{+*},\left(\varepsilon\left(t_{k}\right)\right)_{k}$ converges strongly towards zero in $X$.

From Lemma 3.1 the trajectories of $\hat{x}$ and $\varepsilon$ are precompact in $X$. So from Lemma 7.3 it follows that the functions $\hat{x}$ and $\varepsilon$ are strongly equicontinuous on $\left[0,+\infty\left[\right.\right.$. Consequently, if $\left(\tau_{k}\right)_{k}$ is given as in Definition 5.3, the classical Ascoli-Arzelà Theorem ensures that the sequences $\left(\hat{x}_{\left[\tau_{k}\right]}^{T}\right)_{k}$ and $\left(\varepsilon_{\left[\tau_{k}\right]}^{T}\right)_{k}$ are precompact in $\mathbb{C}([0, T], X)$. So the proofs of Lemma 7.4 and Theorem 5.1 (step a)) run with $\mathcal{C}_{T}=\mathbb{C}([0, T], X)$. In addition since the trajectory of $\varepsilon$ is precompact the weak convergence of $\left(\varepsilon_{\left[\tau_{k}\right]}^{T}\right)_{k}$ becomes strong. Now since $\|\varepsilon\|$ decreases on $\left[0,+\infty\left[\right.\right.$, we have $\varepsilon\left(t_{k}\right) \longrightarrow 0$ and the proof is done.

\subsection{Proof concerning the Cauchy problem $\mathrm{CP}_{\mathbf{F}}\left(\mathrm{x}^{0}\right)$}

Proof of Proposition 3.1. We prove here our fundamental result on the Cauchy problem $C P_{F}\left(x^{0}\right)$, namely Proposition 3.1. We postpone at the end of this Section the proof of the existence result which is done in a particular case in Prüss [31] and can be derived by a standard argument from the Theorem 1 of [13].

Obviously we can restrict the claim of Proposition 3.1 to any compact interval $[0, T]$ with $T>0$. Let us prove first the second assertion, i.e., the integral inequality (4) in $\mathcal{D}^{\prime}(] 0,+\infty[)$ for the solution of $C P_{f}\left(x^{0}\right)$ with $f \in \mathbb{L}_{\text {loc }}^{1}([0,+\infty[, X)$

Let $[x, y]=\lim _{\lambda \searrow 0}((\|x+\lambda y\|-\|x\|) / \lambda)$ be the usual bracket on $X$. If $\Delta=\left(\tau_{0}, \ldots, \tau_{N}\right)$ is a partition of $[0, T]$ and $y$ a function from $[0, T]$ to a set $Y$. Let us denote by $\Delta(y)$ the step function satisfying $\Delta(y)(t)=y\left(\tau_{p}\right)$ when $\left.t \in] \tau_{p-1}, \tau_{p}\right]$ for $p=1, \ldots, N-1$ and $\Delta(y(0))=y\left(\tau_{1}\right), \Delta(y)(t)=y\left(\tau_{N-1}\right)$ for $\left.\left.t \in\right] \tau_{N-1}, \tau_{N}\right]$. Let $x$ be a Duhamel's solution of $S_{f}\left(x^{0}\right)$.

Thanks to the bracket properties, the function $H$ defined by

$$
H(t)=\left(f(t),[x(t), f(t)]_{-}\right)
$$

is Bochner integrable on $[0, T]$. Thus, for this function $H$ there exists an adapted sequence of partitions $\left(\Delta_{n}\right)_{n \in \mathbb{N}}$, that is, for each $n, \Delta_{n}=\left(\tau_{p}^{N_{n}}\right)_{p=0, \ldots, N_{n}}$ is a partition of $[0, T]$ with step size $\varepsilon_{n}$ converging to zero such that the following step functions $H_{n}=\Delta_{n}(H)$ converge towards $H$ in $\mathbb{L}^{1}([0, T], X \times \mathbb{R})$.

Let $x_{n}=\Delta_{n}(x), f_{n}=\Delta_{n}(f), \delta_{p}^{n}=\tau_{p}-\tau_{p-1}, p=1, \ldots, N_{n}$ and for $\tau_{p_{t}-1}^{n}<t \leq \tau_{p_{t}}^{n}$

$$
\gamma_{n}(t)=\frac{1}{\delta_{p_{t}}^{n}} \int_{\tau_{p_{t}-1}^{n}}^{\tau_{p_{t}}^{n}}\left\|f(\tau)-f\left(\tau_{p_{t}}^{n}\right)\right\| \mathrm{d} \tau
$$

We see that $\int_{0}^{T} \gamma_{n}(\tau) \mathrm{d} \tau=\int_{0}^{T}\left\|f(\tau)-f_{n}(\tau)\right\| \mathrm{d} \tau$ has limit zero when $n$ goes to infinity. Thus eventually by extracting a subsequence we can suppose that there is $G \in \mathbb{L}^{1}([0, T])$ satisfying, for all $n \in \mathbb{N}$ and a.a. $t \in[0, T]$,

$$
\left\|f_{n}(t)\right\| \leq G(t) \text {, and } \gamma_{n}(t) \leq G(t)
$$

Let $\varphi \in \mathcal{D}(] 0, T[)$ such that $\varphi \geq 0$. Since $\tau \mapsto\|x(\tau)\| \varphi^{\prime}(\tau)$ is Riemann integrable with compact support on $] 0, T$ [ we have

$$
\begin{aligned}
-\int_{0}^{T}\|x(\tau)\| \varphi^{\prime}(\tau) \mathrm{d} \tau & =-\lim _{n} \sum_{p=1}^{n}\left\|x\left(\tau_{p}^{n}\right)\right\|\left(\varphi\left(\tau_{p}^{n}\right)-\varphi\left(\tau_{p-1}^{n}\right)\right) \\
& =\lim _{n} \sum_{p=1}^{n}\left(\left\|x\left(\tau_{p}^{n}\right)\right\|-\left\|x\left(\tau_{p-1}^{n}\right)\right\|\right) \varphi\left(\tau_{p-1}^{n}\right)
\end{aligned}
$$


Now $x$ being a Duhamel's solution it comes,

$$
x\left(\tau_{p}^{n}\right)=\Phi\left(\tau_{p}^{n}, \tau_{p-1}^{n}\right) x\left(\tau_{p-1}^{n}\right)+\int_{\tau_{p-1}^{n}}^{\tau_{p}^{n}} \Phi\left(\tau_{p}^{n}, \tau\right) f(\tau) \mathrm{d} \tau
$$

Therefore, from this equality and using the classical properties of the brackets (in particular $\left[\xi_{1}, \xi_{2}+\xi_{3}\right]_{-} \leq$ $\left.\left[\xi_{1}, \xi_{2}\right]+\left[\xi_{1}, \xi_{3}\right]_{-}\right)$, together with the contractive aspect of $\Phi(t, s)$, we have,

$$
\begin{aligned}
\left\|x\left(\tau_{p}^{n}\right)\right\|-\left\|x\left(\tau_{p-1}^{n}\right)\right\|= & {\left[x\left(\tau_{p}^{n}\right), x\left(\tau_{p}^{n}\right)\right]_{-}-\left\|x\left(\tau_{p-1}^{n}\right)\right\| } \\
\leq & {\left[x\left(\tau_{p}^{n}\right), \Phi\left(\tau_{p}^{n}, \tau_{p-1}^{n}\right) x\left(\tau_{p-1}^{n}\right)\right] } \\
& +\left[x\left(\tau_{p}^{n}\right), \int_{\tau_{p-1}^{n}}^{\tau_{p}^{n}} \Phi\left(\tau_{p}^{n}, \tau\right) f(\tau) \mathrm{d} \tau\right]_{-}-\left\|x\left(\tau_{p-1}^{n}\right)\right\| \\
\leq & {\left[x\left(\tau_{p}^{n}\right), \int_{\tau_{p-1}^{n}}^{\tau_{p}^{n}} \Phi\left(\tau_{p}^{n}, \tau\right) f(\tau) \mathrm{d} \tau\right]_{-} . }
\end{aligned}
$$

Introduce the following step functions $g_{n}$ defined by

$$
g_{n}(t)=\frac{1}{\delta_{p}^{n}} \int_{\tau_{p-1}^{n}}^{\tau_{p}^{n}} \Phi\left(\tau_{p}^{n}, \tau\right) f(\tau) \mathrm{d} \tau \text { if } \tau_{p-1}^{n}<t \leq \tau_{p}^{n} p=1, \ldots, N_{n} .
$$

We want to prove that $\left(g_{n}\right)_{n}$ converges towards $f$ in $\mathbb{L}^{1}([0, T], X)$. In the one hand, for $\tau_{p_{t}-1}^{n}<t \leq \tau_{p_{t}}^{n}$, we have

$$
\left\|g_{n}(t)-\frac{1}{\delta_{p_{t}}^{n}} \int_{\tau_{p_{t}-1}^{n}}^{\tau_{p_{t}}^{n}} \Phi\left(\tau_{p_{t}}^{n}, \tau\right) f_{n}(t) \mathrm{d} \tau\right\| \leq \frac{1}{\delta_{p_{t}}^{n}} \int_{\tau_{p_{t}-1}^{n}}^{\tau_{p_{t}}^{n}}\left\|f(\tau)-f\left(\tau_{p_{t}}^{n}\right)\right\| \mathrm{d} \tau=\gamma_{n}(t) .
$$

We have already remarked that $\left(\gamma_{n}\right)_{n}$ converges towards zero in $\mathbb{L}^{1}([0, T])$. On the other hand since

$$
\Phi\left(\tau_{p_{t}}^{n}, \tau\right) f_{n}(t) \longrightarrow f(t) \text { for } \tau \in\left[\tau_{p_{t}-1}^{n}, \tau_{p_{t}}^{n}\right]
$$

for almost all $t \in[0, T]$ when $n$ goes to infinity and since from (60) we have

$$
\left\|\frac{1}{\delta_{p_{t}}^{n}} \int_{\tau_{p_{t}-1}^{n}}^{\tau_{p_{t}}^{n}} \Phi\left(\tau_{p_{t}}^{n}, \tau\right) f_{n}(t) \mathrm{d} \tau\right\| \leq\left\|f_{n}(t)\right\| \leq G(t)
$$

a.e. on $[0, T]$, thanks to the Lebesgue dominated Theorem we deduce

$$
\lim _{n} \int_{0}^{T}\left\|g_{n}(\tau)-f(\tau)\right\| \mathrm{d} \tau=0
$$

The bracket properties give

$$
\begin{aligned}
\int_{0}^{T}\left|\left[x_{n}(\tau), g_{n}(\tau)\right]_{-}-\left[x_{n}(\tau), f_{n}(\tau)\right]_{-}\right| \mathrm{d} \tau & \leq \int_{0}^{T}\left\|g_{n}(\tau)-f_{n}(\tau)\right\| \mathrm{d} \tau \\
& \leq \int_{0}^{T}\left\|g_{n}(\tau)-f(\tau)\right\| \mathrm{d} \tau+\int_{0}^{T}\left\|f(\tau)-f_{n}(\tau)\right\| \mathrm{d} t .
\end{aligned}
$$


According to (64) and (65) we see that the sequence $\left(\left[x_{n}, g_{n}\right]_{-}\right)_{n}$ converges towards $[x, f]_{-}$in $\mathbb{L}^{1}([0, T])$ because the same is true by definition for $\left[x_{n}, f_{n}\right]_{-}$. Then with this last conclusion, the relations $(61,62,63)$ and the positivity of $\varphi$ provide

$$
-\int_{0}^{T}\|x(\tau)\| \varphi^{\prime}(\tau) \mathrm{d} \tau \leq \int_{0}^{T}[x(\tau), f(\tau)]_{-} \varphi(\tau) \mathrm{d} \tau .
$$

That ends the proof of this part.

The first assertion, namely the existence of Duhamel's solutions, is given for instance in Prüss [31] in case $F$ is in addition continuous locally bounded.

In our more general framework we remark that if $\lambda \in[0,1]$, according to (3) and (4), every Duhamel's solution $x$ of $C P_{\lambda F}\left(x^{0}\right)$ on $\left[0, T_{0}\right]$ for $0<T_{0} \leq T$ admits the following bound

$$
\sup _{t \in\left[0, T_{0}\right]}\|x(t)\| \leq\left(\left\|x^{0}\right\|+\int_{0}^{T} g(\tau) \mathrm{d} \tau\right) \exp \left(\int_{0}^{T} g(\tau) \mathrm{d} \tau\right) .
$$

Consequently Theorem 1 in [13] and a standard homotopic continuation argument in the Topological Index Theory guarantee the existence of at least a Duhamel's solutions for $C P_{F}\left(x^{0}\right)$ on $[0, T]$.

\subsection{A precompactness lemma}

Denote by $Q P\left(x^{0}, f, A\right)$ the following quasi-autonomous problem

$$
Q P\left(x^{0}, f, A\right)=\left\{\begin{array}{l}
\dot{x}(t)=A x(t)+f(t), t \geq 0 \\
x(0)=x^{0}
\end{array}\right.
$$

where $A$ is a densely defined $m$-dissipative (nonlinear) operator on the reflexive Banach space $X$ and $f$ a locally integrable function on $[0,+\infty[$. With these notations and assumptions we have the following lemma:

Lemma 7.5. Suppose the resolvent of $A$ to be compact and $f$ uniformly continuous and bounded on $[0,+\infty[$. Then the solution $x$ of $Q P\left(x^{0}, f, A\right)$ has a precompact range if and only if it is uniformly continuous and bounded on $[0,+\infty[$.

More general statements could be found in [14].

Proof. (a) Suppose that the trajectory $x\left(\left[0,+\infty[)\right.\right.$ is precompact. Let $\left(t_{n}\right)_{n}$ and $\left(h_{n}\right)_{n}$ be sequences in $[0,+\infty[$ such that $t_{n} \longrightarrow+\infty$ and $h_{n} \downarrow 0$. Then

$$
\left\|x\left(t_{n}+h_{n}\right)-x\left(t_{n}\right)\right\| \leq\left\|x\left(t_{n}+h_{n}\right)-\mathrm{e}^{h_{n} A} x\left(t_{n}\right)\right\|+\left\|\mathrm{e}^{h_{n} A} x\left(t_{n}\right)-x\left(t_{n}\right)\right\| .
$$

But Bénilan's integral inequalities imply

$$
\left\|x\left(t_{n}+h_{n}\right)-\mathrm{e}^{h_{n} A} x\left(t_{n}\right)\right\| \leq \int_{t_{n}}^{t_{n}+h_{n}}\|f(\tau)\| \mathrm{d} \tau,
$$

and thank to the boundedness of $f$,

$$
\lim _{n}\left\|x\left(t_{n}+h_{n}\right)-\mathrm{e}^{h_{n} A} x\left(t_{n}\right)\right\|=0
$$

Now if we consider a cluster point of the bounded sequence $\left(\left\|\mathrm{e}^{h_{n} A} x\left(t_{n}\right)-x\left(t_{n}\right)\right\|\right)_{n}$ (eventually by extracting a subsequence and using the precompactness of $x([0,+\infty[))$ we can suppose

$$
\lim _{n} x\left(t_{n}\right)=l
$$


Then

$$
\lim _{n}\left\|\mathrm{e}^{h_{n} A} x\left(t_{n}\right)-x\left(t_{n}\right)\right\|=\left\|\mathrm{e}^{0 A} l-l\right\|=0 .
$$

The required uniform continuity follows from $(66,67)$ and $(68)$.

(b) Turn now to the converse and assume that $x$ is uniformly continuous and bounded on $\left[0,+\infty\left[\right.\right.$. Let $\left(f_{n}\right)_{n \geq 1}$ and $\left(x_{n}\right)_{n \geq 1}$ sequences of Lipschitz functions such that

$$
\limsup _{n}\left\|f(t)-f_{n}(t)\right\|=0 \text { and } \limsup _{n}\left\|x(t)-x_{n}(t)\right\|=0
$$

For instance we can choose for $f_{n}$ (resp. $x_{n}$ ) the piecewise linear approximation of $f$ (resp. $x$ ) built on the nodal points $\frac{k}{n}, k \in \mathbb{N}$. The uniform continuity of $f$ and $x$ guarantees the Lipschitz aspect of $f_{n}$ and $x_{n}$ as well as relations (69). Of course the Lipschitz constant relative to $f_{n}$ (resp. $x_{n}$ ) depends on $n$. For such approximations we have $\left\|f_{n}\right\|_{\infty} \leq\|f\|_{\infty}$ (resp. $\left.\left\|x_{n}\right\|_{\infty} \leq\|x\|_{\infty}\right)$, what we suppose in the sequel.

(b1) First suppose $x^{0} \in D(A)$. Let $\omega>0$ and denote by $y_{n}$ the solution of the quasi-autonomous problem $Q P\left(x^{0}, f_{n}+\omega x_{n}, A-\omega I\right)$. We have

$$
\left\|y_{n}(t)\right\| \leq \mathrm{e}^{-\omega t}\left\|x^{0}\right\|+\int_{0}^{t} \mathrm{e}^{-\omega(t-s)}\left\|f_{n}(s)+\omega x_{n}(s)\right\| \mathrm{d} s \leq\left\|x^{0}\right\|+\frac{1}{\omega}\|f\|_{\infty}+\|x\|_{\infty}
$$

for all $t \geq 0$. Moreover, since $x$ is solution of $Q P\left(x^{0}, f+\omega x, A-\omega I\right)$, integral inequalities give

$$
\left\|x(t)-y_{n}(t)\right\| \leq \int_{0}^{t} \mathrm{e}^{-\omega(t-s)}\left\|\left(f-f_{n}\right)(s)+\omega\left(x-x_{n}\right)(s)\right\| \mathrm{d} s \leq \frac{1}{\omega}\left\|f-f_{n}\right\|_{\infty}+\left\|x-x_{n}\right\|_{\infty}
$$

for all $t \geq 0$. Consequently from (69) and (70) we deduce

$$
\lim _{n}\left\|x-y_{n}\right\|_{\infty}=0 .
$$

Let $K_{n}$ be a common Lipchitz constant of $f_{n}$ and $x_{n}$. Applying now Bénilan's integral inequalities with the mild solution $y_{n}$ we find

$$
\left\|y_{n}(h)-x^{0}\right\| \leq \int_{0}^{t} \mathrm{e}^{-\omega(t-s)}\left\|f_{n}(s)+\omega x_{n}(s)-A x_{n}^{0}\right\| \mathrm{d} s \leq h\left(\frac{1}{\omega}\left(\|f\|_{\infty}+\left\|A x^{0}\right\|\right)+\|x\|_{\infty}\right)
$$

then

$$
\begin{aligned}
\left\|y_{n}(t+h)-y_{n}(t)\right\| & \leq \mathrm{e}^{-\omega t}\left\|y_{n}(h)-x^{0}\right\|+\int_{0}^{t} \mathrm{e}^{-\omega(t-s)}\left\|f_{n}(s+h)-f_{n}(s)+\omega\left(x_{n}(s+h)-x_{n}(s)\right)\right\| \mathrm{d} s \\
& \leq h\left(\frac{1}{\omega}\left(\|f\|_{\infty}+\left\|A x^{0}\right\|\right)+\|x\|_{\infty}\right)+\left(\frac{1}{\omega}+1\right) K_{n} h:=L_{n} h
\end{aligned}
$$

for all $t \geq 0, h \geq 0$. Therefore $y_{n}$ is Lipschitz on $\left[0,+\infty\left[\right.\right.$, with Lipschitz constant $L_{n}$. But a Lipschitz mild solution of a quasi-autonomous problem $Q P$ in reflexive Banach space $X$ is a strong one (see for instance [7]). It follows

$$
\left\|A y_{n}(t)\right\| \leq\left\|\frac{\mathrm{d} y_{n}}{\mathrm{~d} t}(t)\right\|+\left\|f_{n}(t)+\omega x_{n}(t)\right\| \leq L_{n}+\|f\|_{\infty}+\omega\|x\|_{\infty}
$$

for a.a. $t \geq 0$. Since $y_{n}$ is bounded and the resolvent of $A$ is compact (72) implies that there is a neglectible set $\Omega \subset\left[0,+\infty\left[\right.\right.$ such that $y_{n}\left(\left[0,+\infty[\backslash \Omega)\right.\right.$ is precompact in $X$. Finally in view of the continuity of $y_{n}$ the range of $y_{n}$ is compact. Owing to (71) the latter conclusion gives that $x$ has a precompact range. Indeed we have the following classical topological lemma: 
Lemma 7.6. Assume $\Gamma$ is a subset of $X$ such that for every $\varepsilon>0$ there exists a precompact subset $\mathcal{K}$ of $X$ satisfying,

for each $\gamma \in \Gamma$. Then $\Gamma$ is relatively compact.

$$
d(\gamma, \mathcal{K})=\inf \{\|\gamma-y\| ; y \in \mathcal{K}\} \leq \varepsilon,
$$

(b2) Now if $x^{0} \in X$ we consider a sequence $x_{n}^{0} \in D(A)$ satisfying $\lim x_{n}^{0}=x^{0}$. If $z_{n}$ stands for the solution of $Q P\left(x_{n}^{0}, f, A\right)$ the dissipativity of $A$ leads to

$$
\lim _{n}\left\|x-z_{n}\right\|_{\infty}=0
$$

Because from part (b1) $z_{n}$ has a precompact range the precompactness of $x([0,+\infty[)$ follows from (73) and Lemma 7.6.

\section{Conclusion}

In this paper we have pointed out in some sense a minimum required to construct Luenberger-like semi-linear observers. We showed that the reflexivity of the state space is deeply linked to our abstract nonlinear framework and that Theorem 5.1 appears in this context as a universal weak principle. An important case (see Sect. 3.2 and Ex. 6.4) of strong convergence of the estimate error has been worked out in Theorem 5.2. The considered applications illustrated this abstract approach and expressed how the question of existence of universal inputs (in infinite dimension) and strong observer (for hyperbolic systems) can be tackled.

\section{Appendix}

\subsection{Reformulation of Assumptions (A3, A4)}

In order to check Assumptions (A) in practice it is convenient to give another formulation of Assumptions (A3) and (A4). We consider the normalized duality mapping on $X^{*}$ defined for all $\varphi \in X^{*}$ by,

$$
J(\varphi)=\left\{x \in X^{* *},\langle x, \varphi\rangle_{X^{* *}, X^{*}}=\|\varphi\|_{X^{*}}^{2}=\|x\|_{X^{* *}}^{2}\right\}
$$

Of course, in the sequel since $X$ is reflexive, $X^{* *}$ will be identified with $X$ by means of the canonical isomorphism. A new formulation of Assumption (A3) is as follows:

(A3') There is a dense subset $\Omega^{*}$ of $X^{*}$ such that

$$
\limsup _{h \downarrow 0}\left\|\Phi_{t \geq 0}^{*}(t+h, t) \varphi-\varphi\right\|_{X^{*}}=0
$$

for all $t \geq 0$ and for all $\varphi \in \Omega^{*}$.

Lemma 9.1. Assumption (A3') is equivalent to (A3).

Proof. The implication $\left(\mathrm{A}^{\prime}\right) \Rightarrow(\mathrm{A} 3)$ is obvious. Let us prove now $(\mathrm{A} 3) \Rightarrow\left(\mathrm{A} 3^{\prime}\right)$. Since the set $\left\{\Phi_{u}(t+h, t) x-x ; t \geq 0, h \geq 0, x \in \Omega_{0}\right\}$ is bounded, the condition (A3) holds if and only if (7) holds for all $\varphi \in X^{*}$. Let $\varphi \in \Omega^{*}$, consider $\varphi_{t, h}=\Phi_{u}^{*}(t+h, t) \varphi-\varphi$ and pick,

$$
x_{t, h} \in J\left(\varphi_{t, h}\right),
$$

where $J$ is the normalized duality mapping defined from $X^{*}$ to $X^{* *} \simeq X$. The family $x_{t, h}$ is clearly bounded since we have $\left\|x_{t, h}\right\|=\left\|\varphi_{t, h}\right\|_{X^{*}} \leq 2\|\varphi\|$. Then from (A3) it comes,

$$
\limsup _{h \downarrow 0}\left\langle x_{t, h}, \varphi_{t, h}\right\rangle=\limsup _{h \downarrow 0}\left\langle\Phi_{t \geq 0}(t+h, t) x_{t, h}-x_{t, h}, \varphi\right\rangle=0 .
$$


According to (76), the following equality $\left\|\varphi_{t, h}\right\|^{2}=\left\langle x_{t, h}, \varphi_{t, h}\right\rangle$ deduced from (75), implies $\lim _{h \downarrow 0}\left\|\varphi_{t, h}\right\|=0$ as required.

Another formulation of (A4) follows:

(A4')The convergence $u_{n} \stackrel{\mathcal{U}_{T}}{\longrightarrow} u_{\infty}$ implies

$$
\Phi_{u_{n}}^{*}(t, s) \varphi \stackrel{X^{*}}{\longrightarrow} \Phi_{u_{\infty}}^{*}(t, s) \varphi
$$

for all $t \geq 0$ and all $\varphi \in X^{*}$.

Lemma 9.2. Assumption (A4') is equivalent to (A4).

Proof. Obviously (A4') implies (A4). For the converse we will proceed as in Lemma 9.1. Assume that (A4) holds and $u_{n} \stackrel{\mathcal{U}_{T}}{\longrightarrow} u_{\infty}$. Similarly to Lemma 9.1 , let $\varphi \in X^{*}$ and $(t, s) \in \Xi \cap[0, T]^{2}$ and choose

$$
x_{n} \in J\left(\Phi_{u_{n}}^{*}(t, s) \varphi-\Phi_{u_{\infty}}^{*}(t, s) \varphi\right) .
$$

We have $\left\|x_{n}\right\| \leq 2\|\varphi\|_{X^{*}}$. Thus $\left(x_{n}\right)_{n}$ is weakly precompact in $X$. Suppose $x_{n_{k}} \stackrel{\mathrm{w}}{\longrightarrow} x_{\infty}$. It follows from (77)

$$
\begin{aligned}
\lim \sup _{k}\left\|\Phi_{u_{n_{k}}}^{*}(t, s) \varphi-\Phi_{u_{\infty}}^{*}(t, s) \varphi\right\|_{X^{*}}^{2} & =\lim \sup _{k}\left\langle x_{n_{k}}, \Phi_{u_{n_{k}}}^{*}(t, s) \varphi-\Phi_{u_{\infty}}^{*}(t, s) \varphi\right\rangle \\
& =\lim \sup _{k}\left\langle\Phi_{u_{n_{k}}}(t, s) x_{n_{k}}, \varphi\right\rangle-\left\langle\Phi_{u_{\infty}}(t, s) x_{\infty}, \varphi\right\rangle
\end{aligned}
$$

This last equality together with Assumption (A4) implies

$$
\lim _{k}\left\|\Phi_{u_{n_{k}}}^{*}(t, s) \varphi-\Phi_{u_{\infty}}^{*}(t, s) \varphi\right\|_{X^{*}}=0
$$

and clearly (by contradiction) this relation holds for all subsequences $\left(n_{k}\right)_{k}$. So (A4') is true and the proof is now complete.

\subsection{General examples covered by assumption (A)}

Our assumptions (A1-A4) have a wide field of applications as illustrated in following various examples.

Example 9.1. The bilinear case.

For all $t \geq 0$, let $\mathcal{A}_{u}(t)=A_{0}+u(t) A_{1}$ where $A_{0}$ is a linear densely defined $m$-dissipative operator in $X$, and $A_{1} \in \mathcal{L}(X)$ is dissipative. We take $\mathcal{U} \subseteq \mathbb{L}^{\infty}\left(\left[0,+\infty[)\right.\right.$ if $A_{1}$ is skew-adjoint and $\mathcal{U} \subseteq \mathbb{L}^{\infty}\left(\left[0,+\infty\left[, \mathbb{R}^{+}\right)\right.\right.$if $A_{1}$ is not skew-adjoint. For $T>0$, we consider on $\mathcal{U}_{T}$ the relative weak*-topology of $\mathbb{L}^{\infty}([0, T])$. Then clearly $(\mathrm{A})$ holds in this case.

Example 9.2. Weighted families of operators.

Let $X=X_{1} \oplus \cdots \oplus X_{m}$, where for $k=1, \ldots, m$, the Banach space $X_{k}$ is reflexive. The space $X$ is endowed with the norm $\|\cdot\|_{X}:=\|\cdot\|_{X_{1}}+\cdots+\|\cdot\|_{X_{m}}$. Consider the family of operators $\mathcal{A}_{u}(t):=u_{1}(t) A_{1} \oplus \cdots \oplus u_{m}(t) A_{m}$, where the operators $A_{k}$ are linear densely defined maximal dissipative operator in $X_{k}$. The space of controls is $\mathcal{U}=\mathcal{U}_{1} \times \cdots \times \mathcal{U}_{m}$, where $\mathcal{U}_{k} \subseteq \mathbb{L}^{\infty}\left(\left[0,+\infty[)\right.\right.$ if $A_{k}$ is skew-adjoint and $\mathcal{U}_{k} \subseteq \mathbb{L}^{\infty}\left([0, T], \mathbb{R}^{+}\right)$if $A_{k}$ is not skew-adjoint. For $T>0$, we consider on $\mathcal{U}_{T}$ the relative weak $k^{*}$-topology of $\mathbb{L}^{\infty}\left(\left[0,+\infty\left[, \mathbb{R}^{m}\right)\right.\right.$. The evolution operator is clearly given by $\Phi_{u}(t, s)=\mathrm{e}^{a_{1}(t, s) A_{1}} \oplus \cdots \oplus \mathrm{e}^{a_{m}(t, s) A_{m}}$ with

$$
a_{k}(t, s)=\int_{s}^{t} u_{k}(\tau) \mathrm{d} \tau, \text { and } u=\left(u_{1}, \ldots, u_{m}\right) .
$$


So (A1) and (A2) hold. Moreover, we have (see Hille-Philipps [23], p. 426 or Bénilan-Crandall-Pazy [7], pp. 83-84)

$$
\Phi_{u}^{*}(t, s)=\mathrm{e}^{a_{1}(t, s) A_{1}^{*}} \oplus \cdots \oplus \mathrm{e}^{a_{m}(t, s) A_{m}^{*}} .
$$

In order to check (A3), due to Lemma 9.1, it suffices to prove (A3'). Firstly, notice that $\Omega^{*}:=\operatorname{Dom}\left(A_{1}^{*}\right) \times \cdots \times$ $\operatorname{Dom}\left(A_{m}^{*}\right)$ is dense in $X^{*}$ (see [23], or [7]). Secondly, $\varphi_{k} \in \operatorname{Dom}\left(A_{k}^{*}\right)$ leads classically to

$$
\left\|\mathrm{e}^{a_{k}(t+h, t) A_{k}^{*}} \varphi_{k}-\varphi_{k}\right\|_{X^{*}} \leq\left|a_{k}(t+h, t)\right|\left\|A_{k}^{*} \varphi_{k}\right\|_{X^{*}}
$$

According to $(78,79)$ and thanks to the uniform integrability of $u_{k}, k=1, \ldots, m$, the inequality $(80)$ yields, for all $\varphi=\left(\varphi_{1}, \ldots, \varphi_{m}\right) \in \Omega^{*}$,

$$
\limsup _{h \downarrow 0}\left\|\Phi_{u}^{*}(t+h, t) \varphi-\varphi\right\|_{X^{*}}=0 .
$$

Clearly, because of (79), Assumption (A4') is satisfied and Lemma 9.2 implies (A4). It thus follows that Assumption (A) holds.

Example 9.3. Non autonomous operators with unbounded perturbations.

Let $\mathcal{A}_{u}(t)=A_{0}+u(t) A_{1}$ where $A_{0}$ and $A_{1}$ are closed dissipative linear operators satisfying the conditions $\overline{\operatorname{Dom}\left(A_{0}\right)}=X$ and $\operatorname{Dom}\left(A_{0}\right) \subseteq \operatorname{Dom}\left(A_{1}\right)$.

We suppose that $A_{1}^{-1}$ is bounded and that $A_{0}$ is m-dissipative. Suppose also that $A_{1} \mathrm{e}^{t A_{0}}$ is a bounded linear operator in $X$ for $t>0$, that the map $t \mapsto A_{1} \mathrm{e}^{t A_{0}}$ is continuous at each $t_{0}>0$ in the norm operator topology, and that $t \mapsto\left\|A_{1} \mathrm{e}^{t A_{0}}\right\|=k(t)$ is locally integrable on $\mathbb{R}^{+}$. See, for instance, the class $\beta\left(A_{0}\right)$ with $A_{0}$ dissipative defined in Hille-Philipps [23] (p. 394). The set of controls is given by $\mathcal{U}:=\mathbb{L}^{\infty}\left(\left[0,+\infty[)\right.\right.$ if $A_{1}$ is skew-adjoint and $\mathcal{U}:=\mathbb{L}^{\infty}\left(\left[0,+\infty\left[, \mathbb{R}^{+}\right)\right.\right.$if $A_{1}$ is not skew-adjoint. We will take the weak topology on $\mathcal{U}_{T}$. Typical examples of such systems are given [19], [24], [23]. See also Applications in Section 6.

We postpone in paragraph 9.3 the verification of Assumption (A). Notice that the classical results in Kato [24] or in Hille-Philipps [23] do not apply directly in this measurable time dependent framework.

Of course our conclusion can be trivially extended to the case of direct sums $\mathcal{A}_{u}(t)=\left(A_{01}+u_{1}(t) A_{11}\right) \oplus \cdots \oplus$ $\left(A_{0 m}+u_{m}(t) A_{1 m}\right)$ in a product of Banach spaces $X=X_{1} \times \cdots \times X_{m}$.

Some examples below or detailed in Section 6 illustrate the notion of compact evolution operators.

Example 9.4. Affine control operators generating compact semigroups.

Let $A$ be a generator of a linear compact $\mathbb{C}^{0}$-semigroup $\mathrm{e}^{\tau A}$ for $\tau>0$ and $u \in \mathbb{L}^{\infty}([0,+\infty[)$ satisfying $u(\tau) \geq \varepsilon>0$ for almost $\tau \geq T_{1}$. Then $\mathcal{A}_{u}(t)=u(t) A$ generates a compact evolution operator given by $\Phi_{u}(t, s)=\mathrm{e}^{[a(t)-a(s)] A}$ with $a(t)=\int_{0}^{t} u(\tau) \mathrm{d} \tau$. Indeed, as in Example 3.1, we have $K(h)=\mathrm{e}^{\varepsilon h A}$ and $L(t, h)=\mathrm{e}^{(a(t+h)-a(t)-\varepsilon h) A}$ for $t \geq T_{1}$.

Example 9.5. Multi-inputs controlled operators generating compact semigroups (see Examples 3.1 or 9.4 above).

Consider the product of reflexive Banach spaces $X=\oplus_{i=1}^{m} X_{i}$, with the norm $\|\cdot\|_{X}=\sum_{i}\|\cdot\|_{X_{i}}$. Let $u=$ $\left(u_{1}, \cdots, u_{m}\right) \in \mathbb{L}^{\infty}\left(\left[0,+\infty\left[; \mathbb{R}^{m}\right)\right.\right.$ be such that $u_{i}(\tau) \geq \varepsilon>0$, for almost $\tau \geq T_{1}$, and $a_{i}(t)=\int_{0}^{t} u_{i}(\tau) \mathrm{d} \tau$ for $i=1, \ldots, m$. Let $A_{i}$ be a generator of a $\mathbb{C}^{0}$-semigroup $\mathrm{e}^{t A_{i}}$ in $X_{i}$. Suppose that $\mathrm{e}^{\sigma A_{i}}, i=1, \ldots, m$, is compact for $\sigma>0$. In this case, the evolution operator $\Phi_{u}(t, s)=\oplus_{i=1}^{m} \mathrm{e}^{\left[a_{i}(t)-a_{i}(s)\right] A_{i}}$ associated with $\mathcal{A}_{u}(t)=\oplus_{i=1}^{m} u_{i}(t) A_{i}$, is compact.

Example 9.6. Unbounded non autonomous operators generating compact semigroups.

Let $\mathcal{A}_{u}(t)=A_{0}+u(t) A_{1}$, where $u \in \mathbb{L}^{\infty}\left(\left[0,+\infty[), A_{0}, A_{1}\right.\right.$ are linear, $\operatorname{Dom}\left(A_{0}\right) \subseteq \operatorname{Dom}\left(A_{1}\right)$ and $\overline{\operatorname{Dom}\left(A_{0}\right)}=X$. Suppose that $A_{0}$ generates a compact semigroup and that $\left(\mathcal{A}_{u}(t)\right)$ generates a contractive 
evolution operator $\Phi_{u}$, satisfying the following relation,

$$
\sup _{x^{0} \in \Omega, t \geq 0}\left\|\Phi_{u}(t+h, t) x^{0}-\mathrm{e}^{h A_{0}} x^{0}\right\| \leq o_{h}(1)
$$

for each bounded subset $\Omega$ of $X$, and $h>0$. Then $\Phi_{u}$ is compact. Indeed, relation (81) implies clearly that $\bigcup_{t \geq 0} \Phi_{u}(t+h, t) \Omega$ is precompact since $\mathrm{e}^{h A_{0}} \Omega$ is. This situation is encountered in Example 9.3.

\subsection{Proof of Assumption (A) for unbounded perturbations}

Let us check assumption (A) for unbounded perturbations in Example 9.3.

Assumption (A1). We start with the following generalized Gronwall's lemma.

Lemma 9.3. Let $0<T, p \in[1,+\infty]$ and consider the following integral inequation,

$$
f(t) \leq h_{1}(t)+\int_{0}^{t} g(t-\tau) f(\tau) \mathrm{d} \tau
$$

with unknown $f$ and data $h_{1} \in \mathbb{L}^{p}\left([0, T], \mathbb{R}^{+}\right), g \in \mathbb{L}^{1}\left([0, T], \mathbb{R}^{+}\right)$. Then (82) has a greatest solution (for the usual partial order of $\left.\mathbb{L}^{p}\left([0, T], \mathbb{R}^{+}\right)\right)$in $\mathbb{L}^{p}\left([0, T], \mathbb{R}^{+}\right)$denoted by $m_{h_{1}, g}=m_{h_{1}}$, and the map $h_{1} \mapsto m_{h_{1}}$ is nondecreasing and Lipschitz-continuous in $\mathbb{L}^{p}\left([0, T], \mathbb{R}^{+}\right)$.

Proof. The proof of this Lemma can be derived easily from Lemma 8.10 of Curtain-Pritchard [16]. The maximal solution $m_{h_{1}}$ is given by,

$$
m_{h_{1}}=h_{1}+\sum_{n=1}^{+\infty}\left(G^{n} h_{1}\right)
$$

with,

$$
\begin{gathered}
g_{1}=g \text { and, } g_{n}(t)=\int_{0}^{t} g(t-s) g_{n-1}(s) \mathrm{d} s \\
\left(G^{n} h_{1}\right)(t)=\int_{0}^{t} g_{n}(t-s) h_{1}(s) \mathrm{d} s .
\end{gathered}
$$

Proof of Assumption (A1) for unbounded perturbations (continued). Let $\delta>0 x^{0} \in X, 0 \leq s \leq t \leq T$ and $x_{\delta}^{0}=\mathrm{e}^{\delta A_{0}} x^{0} \in \operatorname{Dom}\left(A_{0}\right)$. Then define the operator $P$ in the space $\mathbb{C}([0, T], X)$ of continuous $X$-valued functions on $[0, T]$ by

$$
(P x)(t)=A_{1} \mathrm{e}^{(t-s) A_{0}} x_{\delta}^{0}+\int_{s}^{t} u(\tau) A_{1} \mathrm{e}^{(t-\tau) A_{0}} x(\tau) \mathrm{d} \tau .
$$

After computing $\chi(P(\Omega))$ and the modulus of continuity of $P(\Omega)$ for bounded subsets $\Omega$ of $\mathbb{C}([s, T], X)$ a suitable application of the Schauder fixed point theorem gives fixed points of $P$ on $\mathbb{C}\left(\left[s, T_{0}\right], X\right)$ for $T_{0}-s \geq 0$ sufficiently small. Since the generalized Gronwall's Lemma 9.3 provides an a priori upper bound for the fixed points of $P$ on $\left[s, T_{0}\right]$ with $T_{0} \leq T$, it easy to see that there exists at least one fixed point of $P$ in $\mathbb{C}([s, T], X)$. Thus we have

$$
y_{\delta}(t)=A_{1} \mathrm{e}^{(t-s) A_{0}} x_{\delta}^{0}+\int_{s}^{t} u(\tau) A_{1} \mathrm{e}^{(t-\tau) A_{0}} y_{\delta}(\tau) \mathrm{d} \tau .
$$

According to the local integrability on $\mathbb{R}^{+}$of $k(t)=\left\|A_{1} \mathrm{e}^{t A_{0}}\right\|$, and using for instance Lemma 9.3 we see that there is a unique function $y_{\delta}$ solution of $(83)$ in $\mathbb{C}([s, T], X)$. Set now $x_{\delta}(t)=A_{1}^{-1} y_{\delta}(t)$. Since $A_{1}^{-1}$ is linear continuous, it comes from (83)

$$
x_{\delta}(t)=\mathrm{e}^{(t-s) A_{0}} x_{\delta}^{0}+\int_{s}^{t} u(\tau) \mathrm{e}^{(t-\tau) A_{0}} A_{1} x_{\delta}(\tau) \mathrm{d} \tau .
$$


Moreover $x_{\delta}$ in $\mathbb{C}([s, T], X)$ satisfies (84) if and only if $x_{\delta}(t) \in \operatorname{Dom}\left(A_{1}\right)$ for all $t \in[s, T]$ and $y_{\delta}()=.A_{1} x_{\delta}($.$) is$ the solution in $\mathbb{C}([s, T], X)$ of (83), since from the Proposition 3.1 and the dissipativity of $A_{1}$ the equation (84) cannot have more than one continuous solution. In addition this Lemma and the dissipativity of $A_{0}$ show that we have

$$
\left\|x_{\delta}(t)\right\| \leq\left\|x_{\delta}^{0}\right\| \leq\left\|x^{0}\right\| .
$$

Thus since the set $\left.\left.\Omega=\left\{\mathrm{e}^{\delta A_{0}} x^{0} ; \delta \in\right] 0,1\right], x^{0} \in X\right\}$ is dense in $X$, the linear operator defined by

$$
\Phi_{u}(t, s) x_{\delta}^{0}=x_{\delta}(t)
$$

has a unique linear extension to the whole space such that

$$
\left\|\Phi_{u}(t, s) x^{0}\right\| \leq\left\|x^{0}\right\|
$$

for all $x^{0} \in X$.

Now according to the following inequality

$$
\left\|\Phi_{u}(t, s) x^{0}-\Phi_{u}(t, s) \mathrm{e}^{\delta A_{0}} x^{0}\right\| \leq\left\|x^{0}-\mathrm{e}^{\delta A_{0}} x^{0}\right\|
$$

for $0 \leq s \leq t \leq T$, the continuity of $(t, s) \mapsto \Phi_{u}(t, s) x^{0}$ on $\Xi$ comes from the continuity of $(t, s) \mapsto \Phi_{u}(t, s) \mathrm{e}^{\delta A_{0}} x^{0}$ on $\Xi$ for all $\delta>0$.

Let $k(\sigma)=\left\|A_{1} \mathrm{e}^{t A_{0}}\right\|_{\mathcal{L}(X)}$ and $\widetilde{h}(\sigma)=k(\sigma) \sup _{\delta \in[0,1]}\left\|x_{\delta}^{0}\right\|$ and $g(\sigma)=\|u\|_{\infty} k(\sigma)$ for $\left.\left.\sigma \in\right] 0, T-s\right]$. Then Lemma 9.3 gives a function $m_{\tilde{h}, g}=m_{\widetilde{h}}$ satisfying

$$
\left\|y_{\delta}\right\| \leq m_{\widetilde{h}}(.-s) \text { on }[s, T]
$$

for $\delta \in] 0,1]$. Let $\gamma>0$. Set $z_{\delta}(\sigma)=y_{\delta}(s+\sigma)$, and $\Delta_{\delta, \gamma}(\sigma)=\left\|z_{\delta}(\sigma)-z_{\gamma}(\sigma)\right\|$ for $\sigma \in[0, T-s]$. From (83) it comes for $\sigma \in] 0, T-s]$,

$$
\Delta_{\delta, \gamma}(\sigma) \leq k(\sigma)\left\|x_{\delta}^{0}-x_{\gamma}^{0}\right\|+\|u\|_{\infty} \int_{0}^{\sigma} k(\sigma-\tau) \Delta_{\delta, \gamma}(\tau) \mathrm{d} \tau .
$$

So suitable applications of Lemma 9.3 show that the family of continuous functions $\left(y_{\delta}(.)\right)_{\delta>0}$ converges uniformly towards a continuous function $y_{0}$ on each compact subset of $\left.] s, T\right]$ when $\delta$ decreases to zero. Moreover by setting

$$
h_{\delta}(.)=k(\cdot+\delta)\left\|x^{0}\right\| \quad \text { on }[0, T-s] \text { for } \delta \geq 0 .
$$

Lemma 9.3 gives as in $(89),\left\|y_{\delta}\right\| \leq m_{h_{\delta}}(\cdot-s)$ and we deduce

$$
\left\|y_{0}\right\| \leq m_{h_{0}}(\cdot-s)
$$

in $\mathbb{L}^{1}([s, T])$. Consequently, for instance according to (85), the Lebesgue dominated theorem insures that $y_{0}=\lim _{\delta \downarrow 0} y_{\delta}$ in $\mathbb{L}^{1}([s, T], X)$ satisfies the following integral equation

$$
y_{0}(t)=A_{1} \mathrm{e}^{(t-s) A_{0}} x^{0}+\int_{s}^{t} u(\tau) A_{1} \mathrm{e}^{(t-\tau) A_{0}} y_{0}(\tau) \mathrm{d} \tau
$$

in $\mathbb{L}^{1}([s, T], X)$.

Setting $x_{0}=\Phi_{u}(\cdot, s) x^{0}$ it follows from (89) the relation

$$
x_{0}(t)=\mathrm{e}^{(t-s) A_{0}} x^{0}+\int_{s}^{t} u(\tau) \mathrm{e}^{(t-\tau) A_{0}} A_{1} x_{0}(\tau) \mathrm{d} \tau .
$$


We can check from this last conclusions, from the definition of $\Phi_{u}$ and from the closedness of the linear operator $A_{1}$ the following claims

$$
\begin{aligned}
& \Phi_{u}(\tau, s) x^{0} \in \operatorname{Dom}\left(A_{1}\right), \tau>s, \\
& y_{0}(\tau)=A_{1}\left(\Phi_{u}(\tau, s) x^{0}\right), \\
& A_{1}\left(\Phi_{u}(\cdot, s) x^{0}\right) \in \mathbb{L}^{1}([s, T], X) .
\end{aligned}
$$

It is now obvious to show that $\Phi_{u}(\tau, s)$ is an evolution operator and Assumption (A1) is satisfied.

Consider the weak-star topology on the controls and let

$$
u_{n} \stackrel{\mathrm{w}^{*}}{\longrightarrow} u_{\infty} \text { in } \mathbb{L}^{\infty}([0, T])
$$

Similarly to [2] (see also [19]), we are going to prove the continuity of the evolution operator w.r.t. the controls $u$.

Lemma 9.4. Let $u \in \mathcal{U}:=\mathbb{L}^{\infty}\left(\left[0,+\infty[)\right.\right.$, we have $\Phi_{u_{n}}(t, s) x^{0} \rightarrow \Phi_{u_{\infty}}(t, s) x^{0}$ for all $x^{0} \in X$ and $(t, s) \in \Xi$.

Proof. Let us set $x_{n}(t)=\Phi_{u_{n}}(t, s) x^{0}, x_{\infty}(t)=\Phi_{u_{\infty}}(t, s) x^{0}$, and $z_{n}(t)=x_{\infty}(t)-x_{n}(t)$ for $t \in[s, T]$.

Since $x_{n}(t)$ and $x_{\infty}(t)$ are Duhamel's solutions, it comes,

$$
z_{n}(t)=\int_{s}^{t} u_{\infty}(\tau) \mathrm{e}^{(t-\tau) A_{0}} A_{1} x_{\infty}(\tau)-u_{n}(\tau) \mathrm{e}^{(t-\tau) A_{0}} A_{1} x_{n}(\tau) \mathrm{d} \tau .
$$

Thus,

$$
z_{n}(t)=\int_{s}^{t}\left[u_{\infty}(\tau)-u_{n}(\tau)\right] \mathrm{e}^{(t-\tau) A_{0}} A_{1} x_{\infty}(\tau) \mathrm{d} \tau+\int_{s}^{t} u_{n}(\tau) \mathrm{e}^{(t-\tau) A_{0}} A_{1} z_{n}(\tau) \mathrm{d} \tau .
$$

In view of (83) the following equality holds

$$
A_{1} z_{n}(t)=\int_{s}^{t}\left[u_{\infty}(\tau)-u_{n}(\tau)\right] A_{1} \mathrm{e}^{(t-\tau) A_{0}} A_{1} x_{\infty}(\tau) \mathrm{d} \tau+\int_{s}^{t} u_{n}(\tau) A_{1} \mathrm{e}^{(t-\tau) A_{0}} A_{1} z_{n}(\tau) \mathrm{d} \tau .
$$

Then the usual Gronwall's lemma gives

$$
\left\|A_{1} z_{n}(t)\right\| \leq \varepsilon_{n} \exp \int_{s}^{t}\left|u_{n}(\tau)\right|\left\|A_{1} \mathrm{e}^{(t-\tau) A_{0}}\right\| \mathrm{d} \tau
$$

with

$$
\varepsilon_{n}=\sup _{t \in[s, T]}\left|\int_{s}^{T}\left[u_{\infty}(\tau)-u_{n}(\tau)\right] A_{1} \mathrm{e}^{(t-\tau) A_{0}} A_{1} x_{\infty}(\tau) 1_{[s, t]}(\tau) \mathrm{d} \tau\right| .
$$

Using suitable piecewise constant approximations of $\mathrm{e}^{(t-\tau) A_{0}} A_{1} x_{\infty}(\tau)$ (see also [2] or [19]) in the relative strong topology of $\mathbb{L}^{1}([s, T], X)$ in $\mathbb{L}^{\infty}([s, T], X)$ from (92) we deduce (with suitable $t_{n} \in[s, T]$ )

$$
\varepsilon_{n}=\int_{s}^{t_{n}}\left[u_{\infty}(\tau)-u_{n}(\tau)\right] A_{1} \mathrm{e}^{\left(t_{n}-\tau\right) A_{0}} A_{1} x_{\infty}(\tau) \mathrm{d} \tau \rightarrow 0 .
$$

Thus, the relation (93) provides $A_{1} z_{n}(t) \rightarrow 0$ and the continuity of $A_{1}^{-1}$ gives $z_{n}(t) \rightarrow 0$ which ends the proof.

From the dissipativity of $A_{0}$ and $A_{1}$ the relation $\left\|\Phi_{u}(t, s) x^{0}\right\| \leq\left\|x^{0}\right\|$ is clear if $u$ is a step function. But such functions are dense in $\mathbb{L}^{\infty}([s, T])$ equipped with the strong relative topology of $\mathbb{L}^{1}([s, T])$ (and therefore in the weak ${ }^{*}$-topology of $\mathbb{L}^{\infty}([s, T])$ ). And therefore the contractive aspect of the evolution operator (previously established) can be deduced immediately from Lemma 9.4.

Assumption (A2). The control-translation property (A2) is obvious. 
Assumption (A3). Let $\Omega_{1}$ be a bounded subset of $X$. Using (84) it comes for $\varphi \in \Omega^{*}=\operatorname{Dom}\left(A_{0}^{*}\right), x^{0} \in$ $\Omega_{1}$, and $h \in[0, T]$,

$$
\begin{aligned}
\left|\left\langle\Phi_{u}(t+h, t) x^{0}-x^{0}, \varphi\right\rangle\right| & \leq\left|\left\langle x, \mathrm{e}^{h A_{0}^{*}} \varphi-\varphi\right\rangle\right|+\|u\|_{\infty}\|\varphi\| \int_{t}^{t+h}\left\|A_{1} x(\sigma)\right\| \mathrm{d} \tau \\
& \leq h\|x\|\left\|A_{0}^{*} \varphi\right\|+\|u\|_{\infty}\|\varphi\| \int_{t}^{t+h}\left\|A_{1} x(\sigma)\right\| \mathrm{d} \tau
\end{aligned}
$$

According to the last inequality the property (A3) follows from the density of $\Omega^{*}$ in $X^{*}$, the boundedness of the set $\left\{\Phi_{u}(t+h, t) x^{0}-x^{0} ; t, h \geq 0, x^{0} \in \Omega_{1}\right\}$, the inequality (88) and the Lipschitz aspect in $\mathbb{L}^{1}([0, T])$ of $h_{1} \mapsto m_{h_{1}}(\cdot)$.

Assumption (A4). Let $u_{n} \stackrel{\mathrm{w} *}{\longrightarrow} u_{\infty}$ in $\mathbb{L}^{\infty}([0, T])$ and $x_{n}^{0} \stackrel{\mathrm{w}}{\longrightarrow} x_{\infty}^{0}$ in $X$. Let $0 \leq s<t \leq T$. For $n \in \mathbb{N} \cup\{\infty\}$ set $x_{n}(\tau)=\Phi_{u_{n}}(\tau, s) x_{n}^{0}$ and $y_{n}(\tau)=A_{1}\left(x_{n}(\tau)\right), \tau \in[s, T+s]$. Of course, it may happen that $y_{n}$ is not defined at $\tau=s$. In fact, from Assumption (A2) we can suppose $s=0$, by eventually changing $T$ into $T-s$.

Let us remark first that $\left(x_{n}\right)_{n}$ is bounded on $[0, T]$ since by the contractive property of $\Phi_{u}$ we have

$$
\left\|x_{n}(\sigma)\right\| \leq \sup _{k}\left\|x_{k}^{0}\right\|<+\infty
$$

Thus $\left(x_{n}(\sigma)\right)_{n}$ is relatively compact in the reflexive space $X$ for all $\sigma \in[0, T]$. Moreover the sequence of functions $\left(x_{n}\right)_{n}$ is weakly-equicontinuous on $[0, T]$. This point can be easily verified by computing $\sup _{n, \sigma}\left|\left\langle\Phi_{u_{n}}(\sigma+h, \sigma) x_{n}(\sigma)-x_{n}(\sigma), \varphi\right\rangle\right|$ for $\varphi \in X^{*}$, similarly to the proof of Assumption (A3) above, since the boundedness of $\left(x_{n}\right)_{n}$ allows the restriction to a dense subset of $X^{*}$ for the functionals $\varphi$. A direct calculation can be also given in view of the inequality (96) given later.

Consequently by the weak Ascoli-Arzelà Theorem (see Vrabie [36], Th. 1.3.2, p. 10) the sequence $\left(x_{n}(.)\right)_{n}$ is relatively compact in $\mathbb{C}\left([0, T], X_{\mathrm{w}}\right)$. Let $z_{\infty}$ be a cluster point in $\mathbb{C}\left([0, T], X_{\mathrm{w}}\right)$ of $\left(x_{n}(.)\right)_{n}$. In order to simplify the notation we shall write $z_{\infty}=\lim _{n} x_{n}$.

At this stage it remains to prove that we have $z_{\infty}=x_{\infty}$. the main point is to show that $\left(y_{n}\right)_{n}$ is dominated in $\mathbb{L}^{1}([0, T])$. In this goal let us introduce $\widetilde{h}(\xi)=k(\xi) \sup _{n}\left\|x_{n}^{0}\right\|, M=\sup _{n}\left\|u_{n}\right\|_{\infty}$, and

$$
g(\xi)=M k(\xi), \widetilde{m}(\xi)=m_{\widetilde{h}, g}(\xi)
$$

for $\xi \in[0, T]$. We have $\widetilde{h}, \widetilde{m} \in \mathbb{L}^{1}([0, T])$, and by (88)

$$
\left\|y_{n}(\sigma)\right\| \leq \tilde{m}(\sigma)
$$

a.e. $\sigma \in[0, T]$. Let $\varphi \in X^{*}$. Thanks to (90) we have,

$$
\left\langle x_{n}(t), \varphi\right\rangle=\left\langle\mathrm{e}^{(t) A_{0}} x_{n}^{0}, \varphi\right\rangle+\int_{0}^{t} u_{n}(\tau)\left\langle y_{n}(\tau), \mathrm{e}^{(t-\tau) A_{0}^{*}} \varphi\right\rangle \mathrm{d} \tau
$$

The first term of the right hand side of (97) converges clearly towards

$$
\lim _{n}\left\langle\mathrm{e}^{t A_{0}} x_{n}^{0}, \varphi\right\rangle=\left\langle\mathrm{e}^{t A_{0}} x_{\infty}^{0}, \varphi\right\rangle
$$

Let us write

$$
\begin{aligned}
\int_{0}^{t} u_{n}(\tau)\left\langle y_{n}(\tau), \mathrm{e}^{(t-\tau) A_{0}^{*}} \varphi\right\rangle \mathrm{d} \tau= & \int_{0}^{t} u_{n}(\tau)\left\langle x_{n}(\tau), A_{1}^{*} \mathrm{e}^{\delta A_{1}^{*}} \mathrm{e}^{(t-\tau) A_{0}^{*}} \varphi\right\rangle \mathrm{d} \tau \\
& +\int_{0}^{t} u_{n}(\tau)\left\langle y_{n}(\tau),\left(I-\mathrm{e}^{\delta A_{1}^{*}}\right) \mathrm{e}^{(t-\tau) A_{0}^{*}} \varphi\right\rangle \mathrm{d} \tau
\end{aligned}
$$


The first term of the right hand side of (99) converges towards

$$
\int_{0}^{t} u_{\infty}(\tau)\left\langle z_{\infty}(\tau), A_{1}^{*} \mathrm{e}^{\delta A_{1}^{*}} \mathrm{e}^{(t-\tau) A_{0}^{*} \varphi}\right\rangle \mathrm{d} \tau
$$

and we have

$$
\lim _{\delta \downarrow 0} \int_{0}^{t} u_{\infty}(\tau)\left\langle z_{\infty}(\tau), A_{1}^{*} \mathrm{e}^{\delta A_{1}^{*}} \mathrm{e}^{(t-\tau) A_{0}^{*}} \varphi\right\rangle \mathrm{d} \tau=\int_{0}^{t} u_{\infty}(\tau)\left\langle z_{\infty}(\tau), A_{1}^{*} \mathrm{e}^{(t-\tau) A_{0}^{*}} \varphi\right\rangle \mathrm{d} \tau .
$$

But $A_{1}$ being weakly-closed (since it is strongly closed and linear) we can claim $z_{\infty}(\tau) \in \operatorname{Dom}\left(A_{1}\right)$ for $\left.\left.\tau \in\right] 0, t\right]$ and,

$$
\lim _{\delta \downarrow 0} \int_{0}^{t} u_{\infty}(\tau)\left\langle z_{\infty}(\tau), A_{1}^{*} \mathrm{e}^{\delta A_{1}^{*}} \mathrm{e}^{(t-\tau) A_{0}^{*}} \varphi\right\rangle \mathrm{d} \tau=\int_{0}^{t} u_{\infty}(\tau)\left\langle\mathrm{e}^{(t-\tau) A_{0}} A_{1} z_{\infty}(\tau), \varphi\right\rangle \mathrm{d} \tau .
$$

In order to estimate the second term in the right hand side of (99) notice that from (96) it follows

$$
\left|u_{n}(\tau)\left\langle y_{n}(\tau),\left(I-\mathrm{e}^{\delta A_{1}^{*}}\right) \mathrm{e}^{(t-\tau) A_{0}^{*}} \varphi\right\rangle\right| \leq M \widetilde{m}(\tau)\left\|\left(I-\mathrm{e}^{\delta A_{1}^{*}}\right) \mathrm{e}^{(t-\tau) A_{0}^{*}} \varphi\right\|
$$

a.e. $\tau \in[0, T]$. Then the Lebesgue dominated convergence theorem gives for $t \in[0, T]$,

$$
\lim _{\delta \downarrow 0} \lim \sup _{n}\left\|\int_{0}^{t} u_{n}(\tau)\left\langle y_{n}(\tau),\left(I-\mathrm{e}^{\delta A_{1}^{*}}\right) \mathrm{e}^{(t-\tau) A_{0}^{*}} \varphi\right\rangle \mathrm{d} \tau\right\|=0 .
$$

Consequently, from $(99,98,100)$ and (101) we deduce

$$
z_{\infty}(t)=\Phi_{u_{\infty}}(t, s) x_{\infty}^{0}
$$

on $[0, T]$.

The authors want to thank Pr J.P. Gauthier, Pr W. Respondek and professor G. Sallet for stimulating discussions on this work.

\section{REFERENCES}

[1] V. Barbu, Analysis and control of nonlinear infinite dimensional systems. Academic Press, Math. Sci. Engrg. 190 (1993).

[2] J.M. Ball, J.E. Marsden and M. Slemrod, Controllability of distributed bilinear systems. SIAM J. Control Optim. 20 (1982) 575-597.

[3] V. Barbu, Nonlinear semigroups and differential equations in Banach spaces. Noordhoff (1976).

[4] A.S. Besicovitch, Almost periodic functions. Dover, New-York (1954).

[5] P. Bénilan, Equations d'évolution dans un espace de Banach quelconque et applications, Thèse. Univ. Paris-XI, Orsay, France (1972).

[6] P. Bénilan, M.G. Crandall and A. Pazy, Bonnes solutions d'un problème d'évolution semi-linéaire. C. R. Acad. Sci. Paris 306 (1988) 527-530.

[7] P. Bénilan, M.G. Crandall and A. Pazy, Nonlinear evolution equations in Banach spaces. Preprint book (to appear).

[8] H. Bounit and H. Hammouri, Observer design for distributed parameter dissipative bilinear systems. Appl. Math. Comput. Sci. 8 (1998) 381-402.

[9] H. Bounit, Contribution à la stabilisation et à la construction d'observateurs pour une classe de systèmes à paramètres distribués, Thesis. Univ. Claude Bernard, Lyon-I, France (1996).

[10] H. Brezis, Analyse fonctionnelle. Masson, Paris, New-York, Barcelone, Milan, Mexico, Sao Paulo (1987).

[11] N. Carmichael, A.J. Pritchard and M.D. Quinn, State and parameter estimations for nonlinear systems. Appl. Math. Optim. 9 (1982) 133-161.

[12] F. Celle, J.P. Gauthier, D. Kazakos and G. Sallet, Synthesis of nonlinear observers: A harmonic analysis approach. Math. System Theory 22 (1989) 291-322. 
[13] J.-F. Couchouron and M. Kamenski, An abstract topological point of view and a general averaging principle in the theory of differential inclusions. Nonlinear Anal. 42 (2000) 1101-1129.

[14] J.-F. Couchouron, Compactness theorems for abstract evolution problems (submitted).

[15] M.G. Crandall, Nonlinear semigroups and evolution governed by accretive operators. Proc. Symp. Pure Math. 45 (1986) 305-337.

[16] R.F. Curtain and A.J. Pritchard, Infinite dimensional linear systems theory. Springer-Verlag, New York (1978).

[17] D. Dochain, Contribution to the analysis and control of distributed parameter systems with application to (bio)chemical processes and robotics, Thesis. Univ. Cath. Louvain, Belgium (1994).

[18] S. Dolecki and L. Russel, A general theory of observation and control. SIAM J. Control Optim. 15 (1977) 185-220.

[19] N. El Alami, Analyse et commande optimale des systèmes bilineaires à paramètres distribués - Application aux procédés énergétiques, Thèse. Univ. de Perpignan, France (1986).

[20] J.P. Gauthier and I. Kupka, Observability and observers for nonlinear systems. SIAM J. Control Optim. 32 (1994) $975-994$.

[21] J.P. Gauthier and I. Kupka, Observability for systems with more outputs than inputs and asymptotic observers. Math. Z. 223 (1996) 47-78.

[22] J.P. Gauthier, C.Z. Xu and A. Bounabat, An observer for infinite dimensional skew-adjoint bilinear systems. J. Math. Syst. Estim. Control 5 (1995) 1-20.

[23] E. Hille and R.S. Philipps, Functional analysis and semi-groups. AMS colloquium publications, Vol. XXXI (1965).

[24] T. Kato, Perturbation theory of linear operators. Springer-Verlag, New-York (1966).

[25] T. Kato, Nonlinear evolution equations in Banach spaces. Proc. of Symp. Appl. Math. 17 (1965) 50-67.

[26] P. Ligarius, J.P. Gauthier and C.Z. Xu, A simple observer for distributed systems: Application on a heat exchanger. J. Math. Systems Estim. Control 8 (1998) 1-23 (retrieval code: 73494).

[27] P. Ligarius, Observateurs de systèmes bilinéaires à paramètres répartis - Applications à un échangeur thermique, Thesis. Univ. of Rouen, France (1995).

[28] A. Pazy, Semigroups of Linear Operators and Applications to Partial Differential Equations. Springer-Verlag, New-York (1983).

[29] A. El Jai and A.J. Pritchard, Capteurs et actionneurs dans l'analyse des systèmes distribués. Masson (1986).

[30] A.J. Pritchard, Introduction to semigroup theory. Springer-Verlag, Lecture Notes in Control Inform. Sci. 185 (1993) 1-22.

[31] J. Prüss, On semilinear evolution equations in Banach spaces. J. Reine Angew. Math. 303/304 (1978) 144-158.

[32] M. Slemrod, Feedback stabilization of a linear control system in Hilbert space with a priori bounded control. Math. Control Signal Syst. 2 (1989) 265-285.

[33] H.J. Sussman, Single input observability of continuous time systems. Math. Systems Theory 12 (1979) 371-393.

[34] E. Sontag, On the observability of polynomial systems. SIAM J. Control Optim. 17 (1979) 139-151.

[35] R. Temam, Infinite dimensional dynamical systems in mechanics and physics. Springer-Verlag, New York, Appl. Math. Sci. (1988).

[36] I.I. Vrabie, Compactness methods for nonlinear evolutions. John Wiley \& Son, Pitman Monogr. Surveys Pures Appl. Math. 32 (1987).

[37] W.M. Wonham, .Linear multivariable control, a geometric approach, 3rd Edn. Springer-Verlag, New York (1985).

[38] C.Z. Xu, P. Ligarius and J.P. Gauthier, An observer for infinite dimensional dissipative bilinear systems. Comput. Math. Appl. 29 (1995) 13-21.

[39] C.Z. Xu and J.P. Gauthier, Analyse et commande d'un échangeur thermique à contre-courant. RAIRO APII 25 (1991) 377-396.

[40] C.Z. Xu, Exact observability and exponential stability of infinite dimensional bilinear systems. Math. Control Signals Syst. 9 (1996) 73-93. 